\title{
Adaptive Planar Curve Tracking Control and Robustness Analysis under State Constraints and Unknown Curvature*
}

\author{
Michael Malisoff $^{\mathrm{a}}$ and Robert Sizemore ${ }^{\mathrm{a}}$ and Fumin Zhang ${ }^{\mathrm{b}}$ \\ ${ }^{a}$ Department of Mathematics, Louisiana State University, Baton Rouge, LA 70803-4918, USA \\ ${ }^{\mathrm{b}}$ Georgia Tech School of Electrical and Computer Engineering, 777 Atlantic Dr. NW, Atlanta, GA 30332-0250, USA
}

\begin{abstract}
We provide adaptive controllers for curve tracking in the plane, under unknown curvatures and control uncertainty, which is a central problem in robotics. The system dynamics include a nonlinear dependence on the curvature, and are coupled with an estimator for the unknown curvature, to form the augmented error dynamics. We prove input-to-state stability of the augmented error dynamics with respect to an input that is represented by additive uncertainty on the control, under polygonal state constraints and under suitable known bounds on the curvature and on the control uncertainty. When the uncertainty is zero, this gives tracking of the curve and convergence of the curvature estimate to the unknown curvature. Our curvature identification result is a significant improvement over earlier results, which do not ensure parameter identification, or which identify the control gain but not the curvature.
\end{abstract}

Key words: adaptive systems, constrained parameters, curve tracking, robotics, robustness, uncertainty

\section{Introduction}

This paper continues our quest (begun in Malisoff et al. (2012), Malisoff and Zhang (2013), and Malisoff and Zhang (2015)) for curve tracking controls that ensure key stability properties under uncertainties and state constraints. Curve tracking is important for navigating mobile robots; see Lumelsky and Stepanov (1987), Malisoff and Zhang (2013), and Woolsey and Techy (2009). The work Micaelli and Samson (1993) provides feedback controls for wheeled mobile robots which track boundaries of obstacles, based on Frenet-Serret frames; and Aguiar and Hespanha (2007), Borhaug and Pettersen (2005), and Do et al. (2004) provide generalized adaptive robot controllers for under-actuated autonomous ships and other cases. See also Justh and Krishnaprasad (2005), Morin and Samson (2008), and Zhang et al. (2007).

Using the curve tracking controls from Zhang et al. (2004b)

\footnotetext{
* Running title: Adaptive curve tracking under unknown curvature. Corresponding author: F. Zhang (Fax: +1-404-8944641, Phone: +1-404-385-2751). A preliminary version appeared in the Proceedings of the 2016 American Control Conference; see the end of Section 1 below for a comparison between the conference version and this paper. Malisoff and Sizemore were supported by NSF-ECCS Grant 1408295. Zhang was supported by ONR Grants N00014-10-10712 (YIP) and N00014-141-0635, and NSF Grants ECCS-0841195, ECCS-0845333 (CAREER), OCE-1032285, IIS-1319874, and CMMI-1436284.

Email addresses: malisoff@lsu.edu (Michael Malisoff), rsizem2@lsu.edu (Robert Sizemore), fumin@gatech.edu (Fumin Zhang).
}

and polygonal state constraints, the work Malisoff et al. (2012) proved robustness of the curve tracking. While experimental evidence of robustness of curve tracking controls had been observed in farming, obstacle avoidance in corridors, ocean sampling, and ship control (in Lenain et al. (2006), Zhang et al. (2004a), Zhang et al. (2007), and Do and Pan (2006), respectively), the work Malisoff et al. (2012) provided a mathematical analysis based on a new Lyapunov function design and therefore provided theoretical justification for the experimental observations. In addition to the Lyapunov analysis, a key contribution in Malisoff et al. (2012) was a proof of robust forward invariance of a class of hexagonal regions $H \subseteq \mathbb{R}^{2}$. For each such $H$, Malisoff et al. (2012) computed the supremum of the set of all constants $\delta_{H}>0$ such that all trajectories starting in $H$, for all additive control uncertainties that are bounded by $\delta_{H}$, remain in $H$. By viewing the planar workspace as a nested sequence $\left\{H_{i}\right\}$ of polygonal regions, this gave predictable tolerance and safety bounds by proving input-tostate stability (ISS) (as defined in Khalil (2002)) of the curve tracking dynamics on each set $H_{i}$, under additive uncertainty on the control and maximal perturbation bounds.

The controls from Malisoff et al. (2012) and Zhang et al. $(2004 b)$ were implemented in our team's deployment of marine robots at Grand Isle, Louisiana, which surveyed the long term impacts of the 2010 Deepwater Horizon oil spill disaster; see Mukhopadhyay et al. (2014). Robust forward invariance can incorporate obstacles that occur in marine surveys. Our work at Grand Isle tested our controls under different control gains, and Malisoff and Zhang (2013) extended Malisoff et al. (2012) by proving adaptive tracking 
with identification of control gains. While our approach does not explicitly take side slip into account, our experimental deployments showed good performance of our robust control approach, even if there are no actuators that compensate for side slip. However, Malisoff et al. (2012), Malisoff and Zhang (2013), and Malisoff and Zhang (2015) assume that we know the curvatures along the curves being tracked, which can make the work difficult to apply. For instance, in marine robotic surveys, the boundary curves of our regions of interest (e.g., areas of pollution shortly after an oil spill) may be uncertain. See Bresch-Pietri and Krstic (2010), Bresch-Pietri and Krstic (2014), and Krstic (2009) for more motivation for adaptiveness.

Therefore, this work provides a new adaptive controller for curve tracking. The controller uses a strict Lyapunov function from Malisoff et al. (2012). However, the novel features of this work include (a) our new dynamical extension that identifies unknown curvatures, (b) our proof of global asymptotic stability of the augmented tracking and curvature identification error dynamics, which is based on a new strict Lyapunov function construction for the augmented error dynamics that gives tracking under constant curvatures, and (c) our robust forward invariance approach to ensuring that the adaptively controlled dynamics respect certain state constraints. Our analysis from Malisoff and Zhang (2013) does not apply under unknown curvatures, because of the nonlinear dependence of the dynamics on the curvature. This makes our work a significant development that is beyond the scope of Malisoff et al. (2012), Malisoff and Zhang (2013), and Malisoff and Zhang (2015).

This paper also provides several improvements over our preliminary version Malisoff and Zhang (2016) of this work, by also including (i) adaptive results for cases where the curvature is a function of the arc length, (ii) a proof of ISS of the augmented tracking and parameter identification dynamics under additive uncertainty, (iii) an analysis of the effects of scaling certain components of the control, and (iv) our allowing curvatures that take positive and negative values. While Aguiar and Hespanha (2007) and Do et al. (2004) cover more complex dynamics (e.g., for ships, without identifying unknown model parameters), we believe that by covering unknown curvatures and proving robust forward invariance, our work is a valuable theoretical step with the potential for more marine robotic applications, where the boundary curves of interest may be uncertain.

\section{Definitions and Notation}

We use the standard classes of comparison functions $\mathcal{K}_{\infty}$ and $\mathcal{K} \mathcal{L}$ from (Khalil, 2002, Chap. 4). Consider any subset $\mathcal{O}$ of a Euclidean space and any point $\mathcal{E} \in \mathcal{O}$. We use the usual definitions of positive definiteness with respect to $\mathcal{E}$, and moduli and nonstrict and strict Lyapunov functions with respect to $(\mathcal{E}, \mathcal{O})$; see Malisoff et al. (2012) for their definitions. Let $|p|_{\mathcal{E}}=|p-\mathcal{E}|$ be the distance between any point $p \in \mathcal{O}$ and $\mathcal{E}$ in the usual Euclidean metric.

Let $\mathcal{U}$ be any subset of a Euclidean space such that $0 \in \mathcal{U}$.
Let $|f|_{\mathcal{S}}$ denote the essential supremum of any function $f$ over any set $\mathcal{S}$, and $|f|_{\infty}$ denote its essential supremum over its entire domain. Take any forward complete system

$$
\dot{x}=\mathcal{F}(x, \delta)
$$

with state space $\mathcal{O}$ and measurable essentially bounded disturbances $\delta:[0, \infty) \rightarrow \mathcal{U}$, where $\mathcal{F}: \mathcal{O} \times \mathcal{U} \rightarrow \mathcal{O}$ satisfies the standard existence and uniqueness of solutions properties and $\mathcal{F}(\mathcal{E}, 0)=0$. Let $\mathcal{S} \subseteq \mathcal{O}$ be a neighborhood of $\mathcal{E}$. We say that the system is input-to-state stable (ISS) with respect to $(\mathcal{U}, \mathcal{E}, \mathcal{S})$ provided there are $\beta \in \mathcal{K} \mathcal{L}$ and $\gamma \in \mathcal{K}_{\infty}$ and a modulus $\Lambda$ with respect to $(\mathcal{E}, \mathcal{S})$ such that

$$
\left|x\left(t, x_{0}, \delta\right)\right|_{\mathcal{E}} \leq \beta\left(\Lambda\left(x_{0}\right), t\right)+\gamma\left(|\delta|_{[0, t]}\right)
$$

holds for all $t \geq 0$, all solutions $x\left(t, x_{0}, \delta\right)$ of (1) with initial states $x_{0} \in \mathcal{S}$, and all $\mathcal{U}$-valued $\delta$ 's. This agrees with the usual ISS condition when $\mathcal{O}=\mathcal{S}=\mathbb{R}^{n}, \mathcal{E}=0$, and $\Lambda(x)=|x|$. The special case where $\mathcal{F}$ only depends on $x$ and $\gamma\left(|\delta|_{[0, t]}\right)$ in (2) is not present is called global asymptotic stability $(G A S)$ with respect to $(\mathcal{E}, \mathcal{S})$. A set $\mathcal{H} \subseteq \mathcal{O}$ is called robustly forwardly invariant for (1) with disturbances valued in $\mathcal{U}$ provided all solutions of (1) with initial states in $\mathcal{H}$ for all disturbances $\delta$ valued in $\mathcal{U}$ remain in $\mathcal{H}$, i.e., $x(t, \mathcal{H}, \delta) \subseteq \mathcal{H}$ for all $t \geq 0$ and all $\mathcal{U}$-valued $\delta$ 's. We let $\partial \mathcal{O}$ denote the boundary of any subset $\mathcal{O}$ of a Euclidean space.

\section{Review of Model and Nonadaptive Cases}

The curve tracking dynamics can be simplified to

$$
\dot{\rho}=-\sin (\phi), \quad \dot{\phi}=\frac{\kappa \cos (\phi)}{1+\kappa \rho}-u_{0}+\Delta
$$

where $\rho$ is the distance between the robot and the closest point on the curve being tracked (which we assume is unique), $\phi$ is the bearing, $\kappa$ is the curvature at the closest point on the curve, $u_{0}$ is the steering control, the real valued essentially bounded function $\Delta$ represents (control, measurement, or model) uncertainty, and the state space is $\mathcal{X}=(0, \infty) \times(-\pi / 2, \pi / 2)$, i.e., $(\rho, \phi)$ takes its values in $\mathcal{X}$; the model was originally derived in Zhang et al. (2004b).

For cases where $\Delta=0$, the work Zhang et al. (2004b) designed a feedback to achieve asymptotic stabilization of an equilibrium corresponding to a constant distance ( $\rho=$ $\left.\rho_{0}>0\right)$ and zero bearing $(\phi=0)$, which occurs when the robot moves parallel to the curve. Since $\kappa$ in Malisoff et al. (2012) was assumed to be known, it used the control

$$
u_{0}=\frac{\kappa \cos (\phi)}{1+\kappa \rho}-h^{\prime}(\rho) \cos (\phi)+\mu \sin (\phi)
$$

where $\mu>0$ is a steering constant, under this assumption:

Assumption 1 The function $h:(0, \infty) \rightarrow[0, \infty)$ is $C^{2}$, there are only finitely many values $\rho$ where $h^{\prime}(\rho)=0$, $\lim _{\rho \rightarrow 0^{+}} h(\rho)=\lim _{\rho \rightarrow \infty} h(\rho)=\infty$, and there is a constant $\rho_{0}>0$ such that $h\left(\rho_{0}\right)=0$. Also, there exist an increasing $C^{1}$ function $\gamma:[0, \infty) \rightarrow[\mu, \infty)$ and a function $\Gamma \in \mathcal{K}_{\infty} \cap C^{1}$ such that $\gamma(h(\rho)) \geq 1+0.5 \mu^{2}+h^{\prime \prime}(\rho)$ and $\Gamma(h(\rho)) \geq\left(h^{\prime}(\rho)\right)^{2}$ hold for all $\rho>0$. Finally, $h^{\prime}(\rho)\left(\rho-\rho_{0}\right)$ is positive for all $\rho>0$ except for $\rho=\rho_{0}$, and $h^{\prime \prime}\left(\rho_{0}\right)>0 . \square$ 


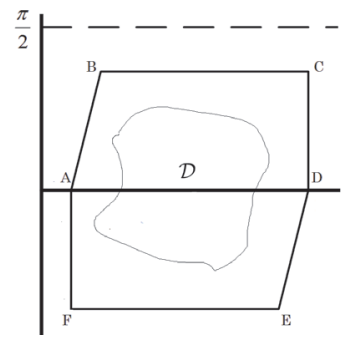

Fig. 1. Hexagon $H\left(\rho_{*}, \mu, K\right)$

Then, for any constant $L>0$, Malisoff et al. (2012) shows that the unperturbed closed loop dynamics

$$
\dot{\rho}=-\sin (\phi), \quad \dot{\phi}=h^{\prime}(\rho) \cos (\phi)-\mu \sin (\phi),
$$

admits the strict Lyapunov function

$$
\begin{aligned}
U(\rho, \phi)= & -h^{\prime}(\rho) \sin (\phi)+\frac{1}{\mu} \int_{0}^{V(\rho, \phi)} \gamma(m) \mathrm{d} m \\
& +L \Gamma(V(\rho, \phi))+\frac{1}{2 L} V(\rho, \phi)
\end{aligned}
$$

with respect to $\left(\left(\rho_{0}, 0\right), \mathcal{X}\right)$, where

$$
V(\rho, \phi)=-\ln (\cos (\phi))+h(\rho) .
$$

The function (7) was used in Zhang et al. (2004b) to prove global asymptotic stability of $(5)$ to $\left(\rho_{0}, 0\right)$, using LaSalle invariance and the fact that $(d / d t) V(\rho(t), \phi(t))=$ $-\mu \sin ^{2}(\phi) / \cos (\phi)$ holds along all solutions of $(5)$ in $\mathcal{X}$.

A key advantage of Malisoff et al. (2012) is that it proves that along all trajectories of (5) in $\mathcal{X}$, the function (6) satisfies $U \geq V$ and the strict Lyapunov decay condition

$$
\dot{U} \leq-0.5\left[h^{\prime}(\rho) \cos (\phi)\right]^{2}-\mathcal{G}(V(\rho, \phi)) \sin ^{2}(\phi)
$$

where $\mathcal{G}(r)=1+\mu\left(L \Gamma^{\prime}(r)+1 /(2 L)\right)$. The strict Lyapunov function decay condition (8) allowed us to prove ISS of

$$
\dot{\rho}=-\sin (\phi), \dot{\phi}=h^{\prime}(\rho) \cos (\phi)-\mu \sin (\phi)+\Delta,
$$

under certain restrictions on the norm $|\Delta|_{\infty}$ of the perturbation $\Delta$, when $\kappa$ is a known positive constant.

We also need the following robust forward invariant results from Malisoff et al. (2012). For any constants $\rho_{*} \in\left(0, \rho_{0} / 2\right)$ and $K>1$, let $\mu \in\left(0, \pi /\left(2 \rho_{*}\right)\right)$ be a constant such that

$$
\mu \tan \left(\mu \rho_{*}\right)>\max \left\{\left|h^{\prime}(\rho)\right|: \rho_{*} \leq \rho \leq \rho_{*}+K \rho_{0}\right\}
$$

and $H\left(\rho_{*}, \mu, K\right)$ be the closed region in the $(\rho, \phi)$-plane that is bounded by the hexagon that has the vertices $A=\left(\rho_{*}, 0\right)^{\top}, B=\left(2 \rho_{*}, \mu \rho_{*}\right)^{\top}, C=\left(\rho_{*}+K \rho_{0}, \mu \rho_{*}\right)^{\top}$, $D=\left(\rho_{*}+K \rho_{0}, 0\right)^{\top}, E=\left(K \rho_{0},-\mu \rho_{*}\right)^{\top}$, and $F=$ $\left(\rho_{*},-\mu \rho_{*}\right)^{\top}$. For each compact set $\mathcal{D} \subseteq \mathcal{X}$, we can choose $\rho_{*}, \mu$, and $K$ such that $\mathcal{D} \subseteq H\left(\rho_{*}, \mu, K\right)$; see Fig. 1 . Set $\Delta_{*}=\min \left\{\left|h^{\prime}(\rho) \cos (\phi)\right|:(\rho, \phi)^{\top} \in \mathrm{AB} \cup \mathrm{ED}\right\}$ and $\Delta_{* *}=\min \left\{\left|h^{\prime}(\rho) \cos (\phi)-\mu \sin (\phi)\right|:(\rho, \phi)^{\top} \in \mathrm{BC} \cup \mathrm{EF}\right\}$. Then $\min \left\{\Delta_{*}, \Delta_{* *}\right\}>0$, by (10) and Assumption 1, and Malisoff et al. (2012) and Malisoff and Zhang (2013) prove the following in terms of $U$ from (6):

Lemma 1 Let Assumption 1 hold, $\rho_{*} \in\left(0, \rho_{0} / 2\right)$ and $K>$ 1 and $L>0$ be any constants, and $\mu \in\left(0, \pi /\left(2 \rho_{*}\right)\right)$ satisfy (10). Then: (a) For any constant $\bar{\Delta} \in\left(0, \min \left\{\Delta_{*}, \Delta_{* *}\right\}\right)$, the set $H\left(\rho_{*}, \mu, K\right)$ is robustly forwardly invariant for (9) with disturbances valued in $\mathcal{U}=[-\bar{\Delta}, \bar{\Delta}]$. (b) For each constant $\Delta_{a}>\min \left\{\Delta_{*}, \Delta_{* *}\right\}$, we can find $a$ $\tilde{p} \in \partial\left(H\left(\rho_{*}, \mu, K\right)\right)$ such that the solution of (9) starting at $\tilde{p}$ for one of the constant perturbations $\Delta= \pm \Delta_{a}$ exits $H\left(\rho_{*}, \mu, K\right)$. (c) There is a constant $v_{0}>0$ such that $U(\rho, \phi) \geq v_{0}\left|\left(\rho-\rho_{0}, \phi\right)\right|^{2}$ for all $(\rho, \phi) \in H\left(\rho_{*}, \mu, K\right)$.

Part (b) of Lemma 1 implies that if we use the control (4), then the bound $\min \left\{\Delta_{*}, \Delta_{* *}\right\}$ cannot be enlarged without violating the forward invariance requirement, so the bound is optimal and not conservative (but see Section 6 for larger perturbation bounds under other controls). Finally, we use the following, whose proof consists of Step 3 of (Malisoff and Zhang, 2013, Appendix B):

Lemma 2 Let $\mathcal{X}^{\sharp}$ be a bounded robustly forwardly invariant set for (1) with disturbances $\delta:[0, \infty) \rightarrow\left[-\delta_{*}, \delta_{*}\right]$ that are bounded by some constant $\delta_{*}>0$, where $\mathcal{F}(0,0)=0$. Let $V^{\sharp}: \mathcal{O} \rightarrow[0, \infty)$ be $C^{1}$ on some open set $\mathcal{O}$ containing $\mathcal{X}^{\sharp}$ and admit a constant $\underline{v}>0$, a continuous positive definite function $\alpha_{0}:[0, \infty) \rightarrow[0, \infty)$, a function $\bar{\gamma} \in \mathcal{K}_{\infty}$, and a modulus $\Lambda$ with respect to $\left(0, \mathcal{X}^{\sharp}\right)$ such that $(d / d t) V^{\sharp} \leq$ $-\alpha_{0}\left(V^{\sharp}\right)+\bar{\gamma}(|\delta|)$ and $\underline{v}|x|^{2} \leq V^{\sharp}(x) \leq \Lambda(x)$ hold along all trajectories of (1) starting in $\mathcal{X}^{\sharp}$ for all $\delta:[0, \infty) \rightarrow$ $\left[-\delta_{*}, \delta_{*}\right]$. Then, we can construct functions $\beta^{\sharp} \in \mathcal{K} \mathcal{L}$ and $\gamma^{\sharp} \in \mathcal{K}_{\infty}$ such that $|x(t)| \leq \beta^{\sharp}(\Lambda(x(0)), t)+\gamma^{\sharp}\left(|\delta|_{[0, t]}\right)$ holds along all trajectories of (1) starting in $\mathcal{X}^{\sharp}$ for all choices of $\delta$, so (1) is ISS with respect to $\left(\left[-\delta_{*}, \delta_{*}\right], 0, \mathcal{X}^{\sharp}\right)$.

\section{Main Adaptive Control and Tracking Result}

\subsection{Statement of Result}

We next leverage the results from the preceding section, to study the two dimensional curve tracking dynamics

$$
\dot{\rho}=-\sin \phi, \quad \dot{\phi}=\frac{\kappa \cos \phi}{1+\kappa \rho}-u_{2}+\delta
$$

with unknown constant curvatures $\kappa$ and piecewise continuous unknown disturbances $\delta:[0, \infty) \rightarrow[-\bar{\delta}, \bar{\delta}]$ with a known bound $\bar{\delta} \geq 0$ (but see Section 5 for nonconstant curvatures). The control $u_{2}$ in (11) will differ from (4), since we will no longer assume that $\kappa$ is known. By writing

$$
\frac{\kappa}{1+\kappa \rho}=\frac{\frac{\kappa}{1+\kappa \rho_{0}}}{1+\frac{\kappa}{1+\kappa \rho_{0}}\left(\rho-\rho_{0}\right)}=\frac{\kappa_{0}}{1+\kappa_{0}\left(\rho-\rho_{0}\right)}
$$

where $\rho_{0}>0$ is our desired distance to the curve and

$$
\kappa_{0}=\frac{\kappa}{1+\kappa \rho_{0}},
$$

we will rescale $\kappa$ to replace $\kappa /(1+\kappa \rho)$ by $\kappa_{0} /\left(1+\kappa_{0}(\rho-\right.$ $\left.\left.\rho_{0}\right)\right)$ in (11) and so also in what follows; see Section 5 for motivation for the transformation (12). Later we specify our robustly forwardly invariant sets to ensure that $1+$ $\kappa_{0}\left(\rho-\rho_{0}\right)$ stays positive in our domain of interest.

While $\kappa_{0}$ is unknown, we assume that we know constants $\underline{c}$ and $\bar{c}$ such that $\kappa_{0} \in(\underline{c}, \bar{c})$. We also assume that Assumption 1 holds. In terms of $U$ from (6), we use the estimator

$$
\dot{\hat{\kappa}}_{0}=\left(\hat{\kappa}_{0}-\underline{c}\right)\left(\bar{c}-\hat{\kappa}_{0}\right) \frac{\cos (\phi)}{\left(1+\left(\rho-\rho_{0}\right) \hat{\kappa}_{0}\right)^{2}} \frac{\partial U}{\partial \phi}(\rho, \phi)
$$


for the unknown scaled curvature $\kappa_{0}$. This is valid, because our $U$ in (6) does not depend on $\kappa$, and because later, we specify our robustly forwardly invariant sets in such a way that $1+\left(\rho-\rho_{0}\right) \hat{\kappa}_{0}$ also stays positive. We use

$$
u_{2}=\frac{\hat{\kappa}_{0} \cos (\phi)}{1+\hat{\kappa}_{0}\left(\rho-\rho_{0}\right)}-h^{\prime}(\rho) \cos (\phi)+\mu \sin (\phi)
$$

where $h$ satisfies Assumption 1. This is valid, because in practice, the current bearing $\phi(t)$ can be detected by sensors, but the curvature $\kappa$ (which is $\mathrm{d} \phi / \mathrm{d} s$ in terms of the curve length parameter $s$ ) is difficult to measure accurately.

Applying (12) to (11) and taking a common denominator, we conclude that the closed loop dynamics for the augmented error $\left(\tilde{q}, \tilde{\kappa}_{0}\right)=\left(\tilde{q}_{1}, \tilde{q}_{2}, \tilde{\kappa}_{0}\right)=\left(\rho-\rho_{0}, \phi, \hat{\kappa}_{0}-\kappa_{0}\right)$ are

$$
\left\{\begin{aligned}
\dot{\tilde{q}}_{1}= & -\sin \left(\tilde{q}_{2}\right) \\
\dot{\tilde{q}}_{2}= & h^{\prime}\left(\tilde{q}_{1}+\rho_{0}\right) \cos \left(\tilde{q}_{2}\right)-\mu \sin \left(\tilde{q}_{2}\right) \\
& -\frac{\tilde{\kappa}_{0} \cos (\phi)}{\left(1+\kappa_{0}\left(\rho-\rho_{0}\right)\left(1+\left(\kappa_{0}+\tilde{\kappa}_{0}\right)\left(\rho-\rho_{0}\right)\right)\right.}+\delta \\
\dot{\tilde{\kappa}}_{0}= & \frac{\left(\kappa_{0}+\tilde{\kappa}_{0}-\underline{c}\right)\left(\bar{c}-\kappa_{0}-\tilde{\kappa}_{0}\right)}{\left(1+\left(\rho-\rho_{0}\right) \hat{\kappa}_{0}\right)^{2}} \cos \left(\tilde{q}_{2}\right) \frac{\partial U}{\partial \phi}(\rho, \phi) .
\end{aligned}\right.
$$

Fix any one of the robustly forwardly invariant hexagons $\mathcal{S}=H\left(\rho_{*}, \mu, K\right)$ from Section 3 , and any constant such that $\bar{\Delta} \in\left(0, \min \left\{\Delta_{*}, \Delta_{* *}\right\}\right)$, where $\min \left\{\Delta_{*}, \Delta_{* *}\right\}$ is from Lemma 1. Choosing any functions $\gamma$ and $\Gamma$ that satisfy Assumption 1 and any constant $L \geq 0$, and defining $V$ by (7), we fix any positive constants $\overline{\mathcal{M}}_{1}$ and $\overline{\mathcal{M}}_{2}$ such that

$$
\begin{aligned}
& \overline{\mathcal{M}}_{1} \geq \frac{1}{\mu} \gamma(V(\rho, \phi))+L \Gamma^{\prime}(V(\rho, \phi))+\frac{1}{2 L} \text { and } \\
& \overline{\mathcal{M}}_{2} \geq \frac{\rho-\rho_{0}}{h^{\prime}(\rho)} \max \left\{1, \frac{\rho-\rho_{0}}{h^{\prime}(\rho) \cos ^{2}(\phi)}\right\}
\end{aligned}
$$

hold for all $(\rho, \phi) \in \mathcal{S}$ such that $\rho \neq \rho_{0}$. The constants $\overline{\mathcal{M}}_{1}$ and $\overline{\mathcal{M}}_{2}$ exist by the continuity of $V$ on the compact set $\mathcal{S}$, continuity of $\gamma$ and $\Gamma^{\prime}$, and the facts that $\mu \rho_{*}<\pi / 2$ and $h^{\prime \prime}\left(\rho_{0}\right)>0$, using L'Hopital's Rule to bound $\left(\rho-\rho_{0}\right) / h^{\prime}(\rho)$. Our first adaptive control theorem is as follows (where ISS must be understood as a robustness property of the closed loop system (16) whose input is the disturbance $\delta$ ):

Theorem 1 Let $\mathcal{S}, \bar{\Delta}, \bar{\delta} \in[0, \bar{\Delta}), \rho_{0}, h, U, \overline{\mathcal{M}}_{1}>0$, and $\overline{\mathcal{M}}_{2}>0$ satisfy the above requirements, let $\lambda \in(0,1)$ be any constant, and let the constants $\underline{c}$ and $\bar{c}>\underline{c}$ satisfy

$$
\begin{gathered}
\bar{c}<\underline{c}+\min \left\{\lambda^{2}(\bar{\Delta}-\bar{\delta}), \sqrt{\frac{2 \lambda^{3}}{\overline{\mathcal{M}}_{1}}}, \sqrt{\frac{\lambda^{3}}{\mathcal{M}_{2}\left(2+\overline{\mathcal{M}}_{1}\right)}}\right\}, \\
\bar{c}<\frac{1-\lambda}{\rho_{0}-\rho_{*}}, \text { and } \underline{c}>\frac{\lambda-1}{\rho_{*}+(K-1) \rho_{0}} .
\end{gathered}
$$

Then, (16) is ISS with respect to $\left([-\bar{\delta}, \bar{\delta}], 0, \mathcal{S}^{\sharp}\right)$ where $\mathcal{S}^{\sharp}=$ $\left\{\left(\tilde{q}, \tilde{\kappa}_{0}\right): \tilde{q}+\left(\rho_{0}, 0\right) \in \mathcal{S}, \tilde{\kappa}_{0}+\kappa_{0} \in(\underline{c}, \bar{c})\right\}$.

See also Section 6 for a method for eliminating the requirement (18), based on scaling $h$ and $\mu$ in the control.

\subsection{Key Robust Forward Invariance Lemma}

To prove Theorem 1, we first prove:

Lemma 3 Let the assumptions of Theorem 1 hold. Then for each initial state $\left(\tilde{q}(0), \tilde{\kappa}_{0}(0)\right) \in \mathcal{S}^{\sharp}$ for (16) and each choice of the uncertainty $\delta:[0, \infty) \rightarrow[-\bar{\delta}, \bar{\delta}]$, the solution

$$
\begin{gathered}
\left(\tilde{q}(t), \tilde{\kappa}_{0}(t)\right)=\left(\rho(t)-\rho_{0}, \phi(t), \hat{\kappa}_{0}(t)-\kappa_{0}\right) \text { for (16) satisfies } \\
\min \left\{1+\kappa_{0} \tilde{q}_{1}(t), 1+\left(\kappa_{0}+\tilde{\kappa}_{0}(t)\right) \tilde{q}_{1}(t)\right\} \geq \lambda
\end{gathered}
$$

for all $t \geq 0$ and $(\tilde{q}(t), \tilde{\kappa}(t)) \in \mathcal{S}^{\sharp}$ for all $t \geq 0$, so $(\rho(t), \phi(t))$ stays in $\mathcal{S}$.

Proof: Fix any rectangle $\left[\rho_{\min }, \rho_{\max }\right] \times\left[\phi_{\min }, \phi_{\max }\right] \subseteq$ $(0, \infty) \times(-\pi / 2, \pi / 2)$ such that $\mathcal{S} \subseteq\left(\rho_{\min }, \rho_{\max }\right) \times$ $\left(\phi_{\min }, \phi_{\max }\right)$ and such that

$$
\bar{c}<\frac{1-\lambda}{\rho_{0}-\rho_{\min }} \text { and } \underline{c}>\frac{\lambda-1}{\rho_{\max }-\rho_{0}}
$$

hold. This can be done by choosing $\rho_{\min }<\rho_{*}$ close enough to $\rho_{*}$, choosing $\rho_{\max }$ close enough to $\rho_{*}+K \rho_{0}$, and using the strictness of the inequalities in (19). We first prove that

$$
1+r\left(\rho-\rho_{0}\right) \geq \lambda
$$

holds for all $\rho \in\left[\rho_{\min }, \rho_{\max }\right]$ and all $r \in[\underline{c}, \bar{c}]$. This will give the required lower bounds $(20)$, once we show that $(\tilde{q}(t), \tilde{\kappa}(t)) \in \mathcal{S}^{\sharp}$ for all $t \geq 0$ when $(\tilde{q}(0), \tilde{\kappa}(0)) \in \mathcal{S}^{\sharp}$.

Consider three cases. Case 1: If $r$ and $\rho-\rho_{0}$ have the same sign, then $1+r\left(\rho-\rho_{0}\right) \geq 1>\lambda$. Case 2: If $r \leq 0 \leq \rho-\rho_{0}$, then $\underline{c}<0$, so $(21)$ gives $r\left(\rho-\rho_{0}\right) \geq \underline{c}\left(\rho-\rho_{0}\right) \geq \underline{c}\left(\rho_{\max }-\right.$ $\left.\rho_{0}\right)>\lambda-1$. Case 3: If $r \geq 0 \geq \rho-\rho_{0}$, then (21) gives $r\left(\rho-\rho_{0}\right) \geq r\left(\rho_{\min }-\rho_{0}\right) \geq \bar{c}\left(\rho_{\min }-\rho_{0}\right)>\lambda-1$, since $\rho_{\text {min }}<\rho_{0}$. Next, fix any initial state $\left(\tilde{q}(0), \tilde{\kappa}_{0}(0)\right) \in \mathcal{S}^{\sharp}$ and a $\delta$. Then the existence of the unique maximal solution on some half open interval of the form $\left[0, t_{\max }\right)$ follows from the local Lipschitzness of the right side of (16), and the fact that the denominators in (16) are positive at the initial time (which follows from our analysis of the preceding three cases, and the fact that $\left.\mathcal{S} \subseteq\left(\rho_{\min }, \rho_{\max }\right) \times\left(\phi_{\min }, \phi_{\max }\right)\right)$.

Next, note that $(18)$ gives $\left|\tilde{\kappa}_{0}(t)\right| \leq \bar{c}-\underline{c}<\lambda^{2}(\bar{\Delta}-\bar{\delta})$, so (22) (applied with $r=\kappa_{0}$ and then $r=\hat{\kappa}_{0}$ ) gives

$$
\left|\frac{\tilde{\kappa}_{0}(t) \cos (\phi(t))}{\left(1+\kappa_{0}\left(\rho(t)-\rho_{0}\right)\right)\left(1+\left(\kappa_{0}+\tilde{\kappa}_{0}(t)\right)\left(\rho(t)-\rho_{0}\right)\right)}+\delta(t)\right|<\bar{\Delta}
$$

for all $t$ such that $(\rho(t), \phi(t)) \in\left[\rho_{\min }, \rho_{\max }\right] \times\left[\phi_{\min }, \phi_{\max }\right]$ and $\hat{\kappa}_{0}(t) \in(\underline{c}, \bar{c})$. Suppose that $\left(\tilde{q}(t), \tilde{\kappa}_{0}(t)\right)$ did not remain in $\mathcal{S}^{\sharp}$, for the sake of obtaining a contradiction. We could then find a maximal time $t_{*}$ such that $\left(\tilde{q}(r), \tilde{\kappa}_{0}(r)\right) \in \mathcal{S}^{\sharp}$ for all $r \in\left[0, t_{*}\right]$. In fact, $\left(\rho\left(t_{*}\right), \phi\left(t_{*}\right)\right)$ lies in the boundary $\partial \mathcal{S}$ of $\mathcal{S}$, since the structure of the $\tilde{\kappa}_{0}$ subdynamics in (16) and a uniqueness of solutions argument (which is analogous to (Malisoff and Zhang, 2013, Footnote 2)) ensure that $\tilde{\kappa}_{0}(t)$ cannot reach $-\kappa_{0}+\underline{c}$ or $-\kappa_{0}+\bar{c}$, so $\hat{\kappa}_{0}$ stays in $(\underline{c}, \bar{c})$.

Since $\mathcal{S} \subseteq\left(\rho_{\min }, \rho_{\max }\right) \times\left(\phi_{\min }, \phi_{\max }\right)$ is compact, we can find a constant $\varepsilon>0$ such that the function $\psi:[0, \varepsilon] \rightarrow \mathbb{R}^{3}$ defined by $\psi(t)=\left(\psi_{1}(t), \psi_{2}(t), \psi_{3}(t)\right)=\left(\tilde{q}\left(t_{*}+t\right), \widetilde{\kappa}_{0}\left(t_{*}+\right.\right.$ $t))$ is such that $\left(\psi_{1}(t)+\rho_{0}, \psi_{2}(t)\right)$ starts in $\partial \mathcal{S}$, is valued in $\left[\rho_{\min }, \rho_{\max }\right] \times\left[\phi_{\min }, \phi_{\max }\right]$, and solves $(9)$ on $[0, \varepsilon]$ with

$$
\Delta(t)=-\frac{\tilde{\kappa}_{0}\left(t_{*}+t\right) \cos \left(\psi_{2}(t)\right)}{\left(1+\kappa_{0} \psi_{1}(t)\right)\left(1+\left(\kappa_{0}+\tilde{\kappa}_{0}\left(t_{*}+t\right)\right) \psi_{1}(t)\right)}+\delta\left(t_{*}+t\right)
$$

which satisfies $\max _{t \in[0, \varepsilon]}|\Delta(t)|<\bar{\Delta}$, by (23). Such an $\varepsilon$ exists, by continuity of $\left(\tilde{q}, \tilde{\kappa}_{0}\right)$. Our choice of $\bar{\Delta}$ as a perturbation bound for maintaining forward invariance of $\mathcal{S}$ and the fact that $\psi(t)$ starts in $\mathcal{S}^{\sharp}$ now imply that $\psi$ stays in $\mathcal{S}^{\sharp}$ on $[0, \varepsilon]$, so $\left(\tilde{q}, \tilde{\kappa}_{0}\right)$ stays in $\mathcal{S}^{\sharp}$ on $\left[0, t_{*}+\varepsilon\right]$, contradicting 
the maximality of $t_{*}$. This proves Lemma 3 .

\subsection{Stability Analysis and Curvature Identification}

We complete the proof of Theorem 1 . Using the decay estimate $(8)$ over $\mathcal{S}$ and the fact that $\hat{\kappa}_{0}=\tilde{\kappa}_{0}+\kappa_{0}$ stays in $(\underline{c}, \bar{c})$, it follows that along all trajectories of (16) in its forwardly invariant set $\mathcal{S}^{\sharp}$, the function

$$
U^{\sharp}\left(\rho, \phi, \tilde{\kappa}_{0}\right)=U(\rho, \phi)+\int_{0}^{\tilde{\kappa}_{0}} \frac{\ell}{\left(\ell+\kappa_{0}-\underline{c}\right)\left(\bar{c}-\ell-\kappa_{0}\right)} \mathrm{d} \ell
$$

satisfies

$$
\begin{aligned}
\dot{U}^{\sharp} \leq & -\frac{1}{2}\left(h^{\prime}(\rho) \cos (\phi)\right)^{2}-\mathcal{G}(V(\rho, \phi)) \sin ^{2}(\phi) \\
& -\frac{\partial U}{\partial \phi}(\rho, \phi) \frac{\tilde{\kappa}_{0} \cos (\phi)}{\left(1+\kappa_{0}\left(\rho-\rho_{0}\right)\right)\left(1+\left(\kappa_{0}+\tilde{\kappa}_{0}\right)\left(\rho-\rho_{0}\right)\right)} \\
& +\left(\tilde{\kappa}_{0} \frac{\cos (\phi)}{\left(1+\hat{\kappa}_{0}\left(\rho-\rho_{0}\right)\right)^{2}}+\delta\right) \frac{\partial U}{\partial \phi}(\rho, \phi) \\
= & -\frac{1}{2}\left(h^{\prime}(\rho) \cos (\phi)\right)^{2}-\mathcal{G}(V(\rho, \phi)) \sin ^{2}(\phi) \\
& +\left\{\frac{\partial U}{\partial \phi}(\rho, \phi) \frac{\cos (\phi)\left(-\tilde{\kappa}_{0}^{2}\right)\left(\rho-\rho_{0}\right)}{\left(1+\hat{\kappa}_{0}\left(\rho-\rho_{0}\right)\right)^{2}\left(1+\kappa_{0}\left(\rho-\rho_{0}\right)\right)}\right\} \\
& +\delta \frac{\partial U}{\partial \phi}(\rho, \phi) .
\end{aligned}
$$

By (17) and the facts that $(\rho(t), \phi(t))$ stays in $\mathcal{S}$ and $(\partial V / \partial \phi)(\rho, \phi)=\tan (\phi)$ on $\mathcal{S}$, our choice (6) of $U$ gives

$$
\begin{aligned}
|(\partial U / \partial \phi)(\rho, \phi)| \leq & \mid-h^{\prime}(\rho) \cos (\phi)+(\gamma(V(\rho, \phi)) / \mu \\
& \left.+L \Gamma^{\prime}(V(\rho, \phi))+1 /(2 L)\right) \tan (\phi) \mid \\
\leq & \left|h^{\prime}(\rho) \cos (\phi)\right|+\overline{\mathcal{M}}_{1}|\tan (\phi)|
\end{aligned}
$$

on our robustly forwardly invariant set $\mathcal{S}$.

Hence, we can use (20) and the triangle inequality to upper bound the quantity in curly braces in (25) by

$$
\begin{aligned}
& \frac{\tilde{\kappa}_{0}^{2}}{\lambda^{3}}\left(\left|h^{\prime}(\rho)\left(\rho-\rho_{0}\right)\right| \cos ^{2}(\phi)+\overline{\mathcal{M}}_{1}\left|\sin (\phi)\left(\rho-\rho_{0}\right)\right|\right) \\
\leq & \frac{\tilde{\kappa}_{0}^{2}}{\lambda^{3}}\left(\left|h^{\prime}(\rho)\left(\rho-\rho_{0}\right)\right| \cos ^{2}(\phi)+\frac{\overline{\mathcal{M}}_{1}}{2} \sin ^{2}(\phi)\right. \\
& \left.+\frac{\overline{\mathcal{M}}_{1}}{2}\left(\rho-\rho_{0}\right)^{2}\right) \\
\leq & \frac{\tilde{\kappa}_{0}^{2}}{\lambda^{3}}\left(\overline{\mathcal{M}}_{2}\left(h^{\prime}(\rho) \cos (\phi)\right)^{2}+\frac{\overline{\mathcal{M}}_{1}}{2} \sin ^{2}(\phi)\right. \\
& \left.+\frac{\overline{\mathcal{M}}_{1}}{2} \overline{\mathcal{M}}_{2}\left(h^{\prime}(\rho) \cos (\phi)\right)^{2}\right) .
\end{aligned}
$$

Also, $\left|\tilde{\kappa}_{0}\right|$ is bounded by $\bar{c}-\underline{c},(18)$ implies that

$$
\begin{aligned}
& \frac{1}{\lambda^{3}}(\bar{c}-\underline{c})^{2} \overline{\mathcal{M}}_{2}\left(1+0.5 \overline{\mathcal{M}}_{1}\right)<\frac{1}{2} \text { and } \\
& \frac{1}{\lambda^{3}}(\bar{c}-\underline{c})^{2}\left(\overline{\mathcal{M}}_{1} / 2\right)<1,
\end{aligned}
$$

and the function $\mathcal{G}$ from section 3 is bounded below by 1 .

Hence, (25) and (27) give a constant $\beta_{0}>0$ such that

$$
\begin{aligned}
\dot{U}^{\sharp} \leq & -\frac{1}{2}\left(h^{\prime}(\rho) \cos (\phi)\right)^{2}-\sin ^{2}(\phi) \\
& +\frac{1}{\lambda^{3}}(\bar{c}-\underline{c})^{2}\left(\overline{\mathcal{M}}_{2}\left(1+\frac{\overline{\mathcal{M}}_{1}}{2}\right)\left(h^{\prime}(\rho) \cos (\phi)\right)^{2}\right. \\
& \left.+\frac{\overline{\mathcal{M}}_{1}}{2} \sin ^{2}(\phi)\right)+\delta \frac{\partial U}{\partial \phi}(\rho, \phi) \\
\leq & -\beta_{0}\left(\left(h^{\prime}(\rho) \cos (\phi)\right)^{2}+\sin ^{2}(\phi)\right)+\delta \frac{\partial U}{\partial \phi}(\rho, \phi)
\end{aligned}
$$

holds along all trajectories of (16) in $\mathcal{S}^{\sharp}$, namely,

$$
\beta_{0}=\min \left\{\frac{1}{2}-\frac{(\bar{c}-\underline{c})^{2}}{\lambda^{3}} \overline{\mathcal{M}}_{2}\left(1+\frac{\overline{\mathcal{M}}_{1}}{2}\right), 1-\frac{(\bar{c}-\underline{c})^{2}}{2 \lambda^{3}} \overline{\mathcal{M}}_{1}\right\} .
$$

We next convert $U^{\sharp}$ into a strict Lyapunov function for (16) with respect to $\left(0, \mathcal{S}^{\sharp}\right)$, having the form

$$
V^{\sharp}\left(\tilde{q}, \tilde{\kappa}_{0}\right)=\overline{\mathcal{M}}_{3} U^{\sharp}\left(\rho, \phi, \tilde{\kappa}_{0}\right)+\tilde{q}_{2} \tilde{\kappa}_{0}
$$

for a suitable constant $\overline{\mathcal{M}}_{3}>0$. To this end, we first pick a constant $\overline{\mathcal{G}}_{1}>\bar{c}-\underline{c}$ such that

$$
\begin{aligned}
& \left|\frac{\partial U}{\partial \phi}(\rho, \phi)\right| \leq \overline{\mathcal{G}}_{1},\left|\dot{\tilde{\kappa}}_{0}\right| \leq \overline{\mathcal{G}}_{1}|\tilde{q}|, \\
& \text { and } U^{\sharp}\left(\rho, \phi, \tilde{\kappa}_{0}\right) \geq \frac{\left|\left(\tilde{q}, \tilde{\kappa}_{0}\right)\right|^{2}}{\mathcal{G}_{1}}
\end{aligned}
$$

hold on $\mathcal{S}^{\sharp}$. Such a $\overline{\mathcal{G}_{1}}$ exists by $(20),(26)$, part (c) of Lemma 1 , and nonnegativity of the integral in (24). Then we can use (20), (31), the fact that $h^{\prime}\left(\rho_{0}\right)=0$, the bounds $\left|\tilde{\kappa}_{0}(t)\right| \leq$ $\bar{c}-\underline{c}$ and $\cos (\phi) \geq \cos \left(\mu \rho_{*}\right)>0$, and the triangle inequality to find constants $\overline{\mathcal{G}}_{2}>0$ and $\overline{\mathcal{G}}_{3}>0$ such that on $\mathcal{S}^{\sharp}$, we get

$$
\begin{aligned}
\frac{d}{d t}\left(\tilde{q}_{2} \tilde{\kappa}_{0}\right) \leq & \left(h^{\prime}\left(\tilde{q}_{1}+\rho_{0}\right) \cos \left(\tilde{q}_{2}\right)-\mu \sin \left(\tilde{q}_{2}\right)\right. \\
& \left.-\frac{\tilde{\kappa}_{0} \cos (\phi)}{\left(1+\kappa_{0}\left(\rho-\rho_{0}\right)\right)\left(1+\hat{\kappa}_{0}\left(\rho-\rho_{0}\right)\right)}\right) \tilde{\kappa}_{0} \\
& +\overline{\mathcal{G}}_{1}\left(|\tilde{q}|^{2}+|\delta|\right) \\
\leq & \overline{\mathcal{G}}_{2}\left(\left|\tilde{\kappa}_{0}\right||\tilde{q}|+|\tilde{q}|^{2}\right)-\overline{\mathcal{G}}_{3} \tilde{\kappa}_{0}^{2}+\overline{\mathcal{G}}_{1}|\delta| \\
\leq & \overline{\mathcal{G}}_{2}\left(\frac{\overline{\mathcal{G}}_{2}}{2 \overline{\mathcal{G}}_{3}}|\tilde{q}|^{2}+\frac{\overline{\mathcal{G}}_{3}}{2 \mathcal{G}_{2}} \tilde{\kappa}_{0}^{2}+|\tilde{q}|^{2}\right)-\overline{\mathcal{G}}_{3} \tilde{\kappa}_{0}^{2} \\
& +\overline{\mathcal{G}}_{1}|\delta| \\
= & \overline{\mathcal{G}}_{2}\left(\frac{\overline{\mathcal{G}}_{2}}{2 \overline{\mathcal{G}}_{3}}+1\right)|\tilde{q}|^{2}-\frac{1}{2} \overline{\mathcal{G}}_{3} \tilde{\kappa}_{0}^{2}+\overline{\mathcal{G}}_{1}|\delta| .
\end{aligned}
$$

We can also find a constant $c_{0}>0$ such that

$$
\beta_{0}\left(\left(h^{\prime}(\rho) \cos (\phi)\right)^{2}+\sin ^{2}(\phi)\right) \geq c_{0}|\tilde{q}|^{2}
$$

so $\dot{U}^{\sharp} \leq-c_{0}|\tilde{q}|^{2}+\overline{\mathcal{G}}_{1}|\delta|$ on $\mathcal{S}^{\sharp}$, using (29) and L'Hopital's rule, since $h^{\prime \prime}\left(\rho_{0}\right)>0$ and $\cos (\phi) \geq \cos \left(\mu \rho_{*}\right)>0$ on $\mathcal{S}$.

Hence, the choice (30) of $V^{\sharp}$, with the constant

$$
\overline{\mathcal{M}}_{3}=1+\overline{\mathcal{G}}_{1}+\frac{1}{c_{0}} \overline{\mathcal{G}}_{2}\left(\frac{\overline{\mathcal{G}}_{2}}{2 \overline{\mathcal{G}}_{3}}+1\right),
$$

implies that on $\mathcal{S}^{\sharp}$, we can find a constant $\overline{\mathcal{G}}_{4}>0$ such that

$$
\begin{aligned}
\dot{V}^{\sharp}\left(\tilde{q}, \tilde{\kappa}_{0}\right) & \leq-\overline{\mathcal{M}}_{3} c_{0}|\tilde{q}|^{2}+\frac{d}{d t}\left(\tilde{q}_{2} \tilde{\kappa}_{0}\right)+\overline{\mathcal{G}}_{4}|\delta| \\
& \leq-c_{0}|\tilde{q}|^{2}-\overline{\mathcal{G}}_{2}\left(\frac{\overline{\mathcal{G}}_{2}}{2 \mathcal{G}_{3}}+1\right)|\tilde{q}|^{2}+\frac{d}{d t}\left(\tilde{q}_{2} \tilde{\kappa}_{0}\right)+\overline{\mathcal{G}}_{4}|\delta| \\
& \leq-\underline{v}\left|\left(\tilde{q}, \tilde{\kappa}_{0}\right)\right|^{2}+\left(\overline{\mathcal{G}}_{1}+\overline{\mathcal{G}}_{4}\right)|\delta| \text { and } \\
V^{\sharp}\left(\tilde{q}, \tilde{\kappa}_{0}\right) & \geq \frac{\overline{\mathcal{M}}_{3}\left|\left(\tilde{q}, \tilde{\kappa}_{0}\right)\right|^{2}}{\overline{\mathcal{G}}_{1}}-0.5|\tilde{q}|^{2}-0.5 \tilde{\kappa}_{0}^{2} \\
& \geq \underline{v}\left|\left(\tilde{q}, \tilde{\kappa}_{0}\right)\right|^{2},
\end{aligned}
$$

where $\underline{v}=\min \left\{c_{0}, \overline{\mathcal{G}}_{3} / 2,1 / 2\right\}$, by $(30)-(32)$.

Also, the following variant of an argument from Appendix B in Malisoff and Zhang (2013) provides a positive definite function $\alpha_{0}$ such that

$$
\alpha_{0}\left(V^{\sharp}\left(\tilde{q}, \tilde{\kappa}_{0}\right)\right) \leq \underline{v}\left|\left(\tilde{q}, \tilde{\kappa}_{0}\right)\right|^{2} \text { for all }\left(\tilde{q}, \tilde{\kappa}_{0}\right) \in \mathcal{S}^{\sharp} .
$$

We choose any constant $\varepsilon \in\left(0,0.5 \min \left\{\bar{c}-\kappa_{0}, \kappa_{0}-\underline{c}\right\}\right)$. Then (i) there is a function $\alpha_{1} \in \mathcal{K}_{\infty}$ such that $V^{\sharp}\left(\tilde{q}, \tilde{\kappa}_{0}\right) \leq$ $\alpha_{1}\left(\left|\left(\tilde{q}, \tilde{\kappa}_{0}\right)\right|\right)$ for all $\left(\tilde{q}, \tilde{\kappa}_{0}\right) \in \mathcal{S}^{\sharp}$ such that $\tilde{\kappa}_{0} \in\left[\underline{c}-\kappa_{0}+\right.$ $\left.\varepsilon, \bar{c}-\kappa_{0}-\varepsilon\right]$ and (ii) there is a constant $c_{1}>0$ such that $c_{1} \leq \underline{v}\left|\left(\tilde{q}, \tilde{\kappa}_{0}\right)\right|^{2}$ for all other points $\left(\tilde{q}, \tilde{\kappa}_{0}\right) \in \mathcal{S}^{\sharp}$. Hence, by separately considering the cases (i) and (ii), we conclude that $(36)$ holds with $\alpha_{0}(r)=\min \left\{c_{1}, \underline{v}\left[\alpha_{1}^{-1}(r)\right]^{2}\right\}$. 
This gives

$$
\dot{V}^{\sharp}\left(\tilde{q}, \tilde{\kappa}_{0}\right) \leq-\alpha_{0}\left(V^{\sharp}\left(\tilde{q}, \tilde{\kappa}_{0}\right)\right)+\left(\overline{\mathcal{G}}_{1}+\overline{\mathcal{G}}_{4}\right)|\delta|
$$

along all trajectories of the (16) in $\mathcal{S}^{\sharp}$. Hence, Theorem 1 follows from (35), (37), and Lemma 2.

\section{Discussion on Assumptions and Extensions}

Theorem 1 applies when $\kappa_{0}$ in (16) lies in $(\underline{c}, \bar{c})$, for any constants $\underline{c} \geq 0$ and $\bar{c}>\underline{c}$ satisfying (18)-(19). However, our derivation of (16) was based on the rescaling (12) of the the curvature to replace the denominator $1+\kappa \rho$ in (11) by $1+\kappa_{0}\left(\rho-\rho_{0}\right)$. The rescaling was used to introduce the $\rho-\rho_{0}$ terms in (27), and was also key to ensuring parameter identification, which would not be possible using standard adaptive techniques (which generally provide tracking of the states but not convergence of the parameter estimate to the true parameter value under state constraints). In terms of the curvature parameter $\kappa$ from the original model (11), our bound requirements are then $\underline{c}<\kappa /\left(1+\kappa \rho_{0}\right)<\bar{c}$, by our relation (13) between $\kappa_{0}$ and $\kappa$.

In robotics, curve tracking is usually done for straight lines or circles, or curves whose curvatures change slowly relative to the convergence speed of the robot and so can be regarded as constant. This is analogous to drivers of cars, who prefer roads that do not have large curvatures, and this motivated our assumption of constant curvatures. However, we can generalize our results to allow nonconstant curvatures that can take negative values. For instance, assume that the unknown curvature is some function $\kappa^{\sharp}(s)=\kappa+\eta(s)$ of the curve length $s$ for some constant $\kappa>0$, and that we know a constant $\bar{\delta} \in(0, \kappa)$ such that $\sup _{s}|\eta(s)| \leq \bar{\delta}$. Then replacing $\kappa$ by $\kappa^{\sharp}(s)$ in (11) produces

$$
\dot{\rho}=-\sin \phi, \quad \dot{\phi}=\frac{\kappa \cos \phi}{1+\kappa \rho}-u_{2}+\delta
$$

with the unknown constant nominal curvature $\kappa \geq 0$, where

$$
\delta=\frac{\cos (\phi) \eta(s)}{(1+(\kappa+\eta(s)) \rho)(1+\kappa \rho)}
$$

is bounded by $\bar{\delta}$. Then Theorem 1 ensures ISS with respect to $\delta$ (but see below for another approach under nonconstant $\kappa$ 's, where we can often ensure that $\lim _{t \rightarrow \infty}(\rho(t), \phi(t))=\left(\rho_{0}, 0\right)$, instead of the weaker ISS property). Also, we can satisfy our requirements using $h(\rho)=\alpha\left(\rho-\rho_{0}\right)^{2}$ for any constant $\alpha>0$, by only requiring the conditions from Assumption 1 to hold for all $\rho \in\left[\rho_{*}, \rho_{*}+K \rho_{0}\right]$, since this implies that (8) holds on $\mathcal{S}$, so the requirement $\lim _{\rho \rightarrow 0^{+}} h(\rho)=\infty$ is not needed in $\mathcal{S}$.

Here is one way to allow larger scaled curvature bounds $\bar{c}$. The second inequality in (28) was used to ensure that the term $-\mathcal{G}(V(\rho, \phi)) \sin ^{2}(\phi)$ in $(25)$ had a larger magnitude than $\left(1 / \lambda^{3}\right) \tilde{\kappa}_{0}^{2}\left(\overline{\mathcal{M}}_{1} / 2\right) \sin ^{2}(\phi)$ in $(27)$, where $\mathcal{G}$ is defined in Section 3. If we make the choices of $\gamma$ and $\Gamma$ from Malisoff et al. (2012), then since $\mathcal{G}$ is bounded below by $1+\mu\left(L\left(18 \alpha / \rho_{0}\right)+1 /(2 L)\right)$, it follows that Theorem 1 remains true if we replace the second inequality in (28) by

$$
\frac{1}{\lambda^{3}}(\bar{c}-\underline{c})^{2} \frac{\overline{\mathcal{M}}_{1}}{2}<1+\mu\left(\frac{18 L \alpha}{\rho_{0}}+\frac{1}{2 L}\right) .
$$

Hence, Theorem 1 remains true if we replace $\sqrt{2 \lambda^{3} / \overline{\mathcal{M}}_{1}}$ on the right side of (18) by the larger bound

$$
\sqrt{\frac{2 \lambda^{3}}{\mathcal{M}_{1}}}\left(1+\mu\left(\frac{18 L \alpha}{\rho_{0}}+\frac{1}{2 L}\right)\right)^{1 / 2} .
$$

We next give another approach to enlarging the curvature bound. For an application of Theorem 1, see Section 8.

\section{Effects of Scaling Control Terms}

It is natural to surmise that scaling $\mu>0$ and $h$ in the formula (15) for the control by large enough constants (without scaling the curvature term in (15)) should make it possible to extend our analysis to allow any curvature values for which the denominator in our adaptive control formula (15) is never zero, because it makes the curvature term small relative to the other terms in the control. In this section, we study the effects of such scalings, which allow us to drop the condition (18) on $\bar{c}$ and make it possible to obtain ISS under larger perturbation bounds, and which therefore allow larger sup norms on the nonconstant parts $\eta(s)$ of the curvatures from the previous section; see (39).

To this end, we replace the control $u_{0}$ from (4), the nonstrict Lyapunov function $V$ from (7), and the strictified Lyapunov function $U$ from (6) by their scaled analogs

$$
\begin{aligned}
& u_{s, 0}=\frac{\kappa \cos (\phi)}{1+\kappa \rho}-\mathcal{M}_{*} h^{\prime}(\rho) \cos (\phi)+\mathcal{M}_{*} \mu \sin (\phi), \\
& V_{s}(\rho, \phi)=-\frac{1}{\mathcal{M}_{*}} \ln (\cos (\phi))+h(\rho), \text { and } \\
& U_{s}(\rho, \phi)=-\frac{1}{\mathcal{M}_{*}} h^{\prime}(\rho) \sin (\phi)+\frac{1}{\mu} \int_{0}^{V_{s}(\rho, \phi)} \gamma(m) \mathrm{d} m \\
& \quad+\frac{L}{\mathcal{M}_{*}} \Gamma\left(V_{s}(\rho, \phi)\right)+\frac{1}{2 L} V_{s}(\rho, \phi),
\end{aligned}
$$

respectively, where the constant $\mathcal{M}_{*} \geq 1$ will be chosen, and $L>0$ is a tuning constant as before. We assume that Assumption 1 holds, and that $h^{\prime \prime}$ is nonnegative valued. Before explaining how the scaling constant effects the proof of Theorem 1, we first explain how the preliminary results from Section 3 must be changed to account for $\mathcal{M}_{*}$.

First notice that with the new control $u_{s, 0}$, the closed loop nonadaptive unperturbed dynamics (5) must become

$$
\left\{\begin{array}{l}
\dot{\rho}=-\sin (\phi) \\
\dot{\phi}=\mathcal{M}_{*} h^{\prime}(\rho) \cos (\phi)-\mathcal{M}_{*} \mu \sin (\phi) .
\end{array}\right.
$$

Also, along all solutions of $(43)$ in $(0, \infty) \times(-\pi / 2, \pi / 2)$, we still have the nonstrict Lyapunov function decay condition $\dot{V}=-\mu \sin ^{2}(\phi) / \cos (\phi)$, since we can use the $\mathcal{M}_{*}$ in the formula for $V_{s}$ to cancel the $\mathcal{M}_{*}$ 's in (9). If we choose $N(\rho, \phi)=-h^{\prime}(\rho) \sin (\phi)$, then

$$
\begin{aligned}
\frac{1}{\mathcal{M}_{*}} \dot{N}(\rho, \phi)= & \frac{1}{\mathcal{M}_{*}}\left[h^{\prime \prime}(\rho) \sin ^{2}(\phi)\right. \\
& -h^{\prime}(\rho) \cos (\phi)\left(\mathcal{M}_{*} h^{\prime}(\rho) \cos (\phi)\right. \\
& \left.\left.-\mathcal{M}_{*} \mu \sin (\phi)\right)\right] \\
\leq & h^{\prime \prime}(\rho) \sin ^{2}(\phi)-\frac{1}{2}\left(h^{\prime}(\rho) \cos (\phi)\right)^{2} \\
& +\frac{1}{2} \mu^{2} \sin ^{2}(\phi),
\end{aligned}
$$

holds along all trajectories of (43), by the triangle inequality. Hence, the same argument (from the proof of (Malisoff 
et al., 2012, Theorem 1)) that led to (8) gives

$$
\begin{aligned}
& \dot{U}_{s} \leq-0.5\left[h^{\prime}(\rho) \cos (\phi)\right]^{2}-\mathcal{G}_{s}\left(V_{s}(\rho, \phi)\right) \sin ^{2}(\phi) \\
& \text { and } U_{s}(\rho, \phi) \geq V_{s}(\rho, \phi)
\end{aligned}
$$

along all trajectories of $(43)$ in $(0, \infty) \times(-\pi / 2, \pi / 2)$, where $\mathcal{G}_{s}(r)=1+\mu\left(L \Gamma^{\prime}(r) / \mathcal{M}_{*}+1 /(2 L)\right)$.

We also change the top left and bottom right vertices $B$ and $E$ of our hexagons from Section 3 to

$$
\begin{aligned}
& B=\left(\rho_{*}\left(1+\left(1 / \mathcal{M}_{*}\right)\right), \mu \rho_{*}\right) \text { and } \\
& E=\left(K \rho_{0}+\left(1-\left(1 / \mathcal{M}_{*}\right)\right) \rho_{*},-\mu \rho_{*}\right)
\end{aligned}
$$

respectively, and keep all other vertices unchanged. Then the new slope for the legs $\mathrm{AB}$ and $\mathrm{DE}$ is $\mathcal{M}_{*} \mu$. This changes the supremum $\bar{\Delta}$ for the allowable perturbation bound from $\bar{\Delta}$ to $\mathcal{M}_{*} \bar{\Delta}$, by a similar proof to that of (Malisoff $e t$ al., 2012, Theorem 2), except with the intercept function

$$
\mathcal{I}(\rho, \phi)=\phi-\mu \rho
$$

in the earlier proof replaced by $\mathcal{I}_{s}(\rho, \phi)=\phi-\mathcal{M}_{*} \mu \rho$.

With these changes, we now define $\mathcal{S}, \bar{\Delta}, \bar{\delta} \in(0, \bar{\Delta}), \rho_{0}, h$, $\overline{\mathcal{M}}_{1}$, and $\overline{\mathcal{M}}_{2}$ as before, and the new update law

$$
\dot{\hat{\kappa}}_{0}=\left(\hat{\kappa}_{0}-\underline{c}\right)\left(\bar{c}-\hat{\kappa}_{0}\right) \frac{\cos (\phi)}{\left(1+\left(\rho-\rho_{0}\right) \hat{\kappa}_{0}\right)^{2}} \frac{\partial U_{s}}{\partial \phi}(\rho, \phi) .
$$

We use the preceding estimator $\hat{\kappa}_{0}$ for the scaled curvature $\kappa_{0}$ in the new control

$$
u_{2}=\frac{\hat{\kappa}_{0} \cos (\phi)}{1+\hat{\kappa}_{0}\left(\rho-\rho_{0}\right)}-\mathcal{M}_{*} h^{\prime}(\rho) \cos (\phi)+\mathcal{M}_{*} \mu \sin (\phi),
$$

which produces the augmented error dynamics

$$
\left\{\begin{aligned}
\dot{\tilde{q}}_{1}= & -\sin \left(\tilde{q}_{2}\right) \\
\dot{\tilde{q}}_{2}= & \mathcal{M}_{*} h^{\prime}\left(\tilde{q}_{1}+\rho_{0}\right) \cos \left(\tilde{q}_{2}\right)-\mathcal{M}_{*} \mu \sin \left(\tilde{q}_{2}\right) \\
& -\frac{\tilde{\kappa}_{0} \cos (\phi)}{\left(1+\kappa_{0}\left(\rho-\rho_{0}\right)\right)\left(1+\left(\kappa_{0}+\tilde{\kappa}_{0}\right)\left(\rho-\rho_{0}\right)\right)}+\delta \\
\dot{\tilde{\kappa}}_{0}= & \frac{\left(\kappa_{0}+\tilde{\kappa}_{0}-\underline{c}\right)\left(\bar{c}-\kappa_{0}-\tilde{\kappa}_{0}\right)}{\left(1+\left(\rho-\rho_{0}\right) \hat{\kappa}_{0}\right)^{2}} \cos \left(\tilde{q}_{2}\right) \frac{\partial U_{s}}{\partial \phi}(\rho, \phi) .
\end{aligned}\right.
$$

for the error variable $\left(\tilde{q}, \tilde{\kappa}_{0}\right)=\left(\tilde{q}_{1}, \tilde{q}_{2}, \tilde{\kappa}_{0}\right)=\left(\rho-\rho_{0}, \phi, \hat{\kappa}_{0}-\right.$ $\left.\kappa_{0}\right)$ as before. Then we can prove the following result, which allows us to drop the requirement (18) on $\underline{c}$ and $\bar{c}$ :

Theorem 2 Let $\mathcal{S}, \bar{\Delta}, \rho_{0}, h, \overline{\mathcal{M}}_{1}$, and $\overline{\mathcal{M}}_{2}$ satisfy the above requirements, let $\lambda \in(0,1)$ be any constant, and let the constants $\mathcal{M}_{*} \geq 1, \bar{\delta} \in\left[0, \mathcal{M}_{*} \bar{\Delta}\right), \underline{c}$, and $\bar{c}>\underline{c}$ satisfy

$$
\begin{aligned}
& \mathcal{M}_{*}>\max \left\{\mathcal{M}_{A}, \mathcal{M}_{B}\right\}, \\
& \bar{c}<\frac{1-\lambda}{\rho_{0}-\rho_{*}}, \text { and } \underline{c}>\frac{\lambda-1}{\rho_{*}+(K-1) \rho_{0}},
\end{aligned}
$$

where

$$
\mathcal{M}_{A}=\frac{1}{\Delta}\left(\frac{1-\lambda}{\lambda^{2}}\left(\frac{1}{\rho_{0}-\rho_{*}}+\frac{1}{\rho_{*}+(K-1) \rho_{0}}\right)+\bar{\delta}\right)
$$

and

$$
\begin{aligned}
\mathcal{M}_{B}= & \max \left\{0.5 \overline{\mathcal{M}}_{1}, \overline{\mathcal{M}}_{2}\left(2+\overline{\mathcal{M}}_{1}\right)\right\} \\
& \times \frac{(1-\lambda)^{2}}{\lambda^{3}}\left(\frac{1}{\rho_{0}-\rho_{*}}+\frac{1}{\rho_{*}+(K-1) \rho_{0}}\right)^{2} .
\end{aligned}
$$

Then, (49) is ISS with respect to $\left([-\bar{\delta}, \bar{\delta}], 0, \mathcal{S}^{\sharp}\right)$ where $\mathcal{S}^{\sharp}=$ $\left\{\left(\tilde{q}, \tilde{\kappa}_{0}\right): \tilde{q}+\left(\rho_{0}, 0\right) \in \mathcal{S}, \tilde{\kappa}_{0}+\kappa_{0} \in(\underline{c}, \bar{c})\right\}$.
Proof: We indicate the changes in the proof of Theorem 1 that are needed to prove Theorem 2 . We replace $V$ and $U$ in the proof of Theorem 1 by their scaled versions from (42), and we scale $h^{\prime}\left(\tilde{q}_{1}+\rho_{0}\right) \cos \left(\tilde{q}_{2}\right)-\mu \sin \left(\tilde{q}_{2}\right)$ by $\mathcal{M}_{*}$. Since

$$
\frac{\partial V_{s}(\rho, \phi)}{\partial \phi}=\frac{\tan (\phi)}{\mathcal{M}_{*}},
$$

and since the $-h^{\prime}(\rho) \sin (\phi)$ in the formula for $U$ was scaled by $1 / \mathcal{M}_{*}$ in the formula for $U_{s}$, we can replace $(26)$ by

$$
\left|\frac{\partial U}{\partial \phi}(\rho, \phi)\right| \leq \frac{1}{\mathcal{M}_{*}}\left(\left|h^{\prime}(\rho) \cos (\phi)\right|+\overline{\mathcal{M}}_{1}|\tan (\phi)|\right) .
$$

This lets us scale the left sides of $(28)$ by $1 / \mathcal{M}_{*}$, so since our maximal allowable perturbation bound is now $\mathcal{M}_{*} \bar{\Delta}$, the proof of Theorem 1 shows that its conclusions stay true if

$$
\begin{aligned}
& \bar{c}< \\
& \underline{c}+\min \left\{\lambda^{2}\left(\mathcal{M}_{*} \bar{\Delta}-\bar{\delta}\right), \sqrt{\frac{2 \lambda^{3} \mathcal{M}_{*}}{\mathcal{M}_{1}}}, \sqrt{\frac{\lambda^{3} \mathcal{M}_{*}}{\mathcal{M}_{2}\left(2+\mathcal{M}_{1}\right)}}\right\}, \\
& \bar{c}<\frac{1-\lambda}{\rho_{0}-\rho_{*}} \text { and } \underline{c}>\frac{\lambda-1}{\rho_{*}+(K-1) \rho_{0}}
\end{aligned}
$$

are all satisfied. The theorem now follows because our lower bounds for $\mathcal{M}_{*}$ in (50) and for $\underline{c}$ in (56) imply that

$$
\begin{aligned}
& \underline{c}+\min \left\{\lambda^{2}\left(\mathcal{M}_{*} \bar{\Delta}-\bar{\delta}\right), \sqrt{\frac{2 \lambda^{3} \mathcal{M}_{*}}{\mathcal{M}_{1}}}, \sqrt{\frac{\lambda^{3} \mathcal{M}_{*}}{\overline{\mathcal{M}}_{2}\left(2+\mathcal{M}_{1}\right)}}\right\} \\
& \geq \frac{1-\lambda}{\rho_{0}-\rho_{*}},
\end{aligned}
$$

so we can omit the bound (55), since it follows from (56).

Remark 1 Our proof of Theorem 2 shows that we can omit $1 /\left(\rho_{*}+(K-1) \rho_{0}\right)$ from (51)-(52) when $\kappa_{0}$ is known to be positive, because in that case, we can choose $\underline{c}=0$. When we can scale the steering constant $\mu$ and $h$ in the control, we can sometimes use Theorem 2 to get larger bounds on $\bar{c}$ than what we would get from Theorem 1; see Section 8.

In the next section, we provide another variant of Theorem 1 , which uses an artificial neural network approach to prove curve tracking under uncertain nonconstant curvatures.

\section{Unknown Nonconstant Curvatures}

Theorems 1-2 provide curve tracking and parameter identification under state constraints and control uncertainty. However, Theorems 1-2 require that the scaled curvatures $\kappa_{0}$ (and so also the curvatures $\kappa$ in the original dynamics) be constant, and our ISS extension from Section 5 provides ISS with respect to nonconstant parts $\eta(s)$ of nonconstant curvatures $\kappa^{\sharp}(s)=\kappa+\eta(s)$ with constant parts $\kappa$, without ensuring the curve tracking condition that $\lim _{t \rightarrow \infty}(\rho(t), \phi(t))=\left(\rho_{0}, 0\right)$. In this section, we give a partial extension of Theorem 1 to cases where the scaled curvature $\kappa_{0}$ is again a function of arc length $s$, and where we have an artificial neural network expansion approximation

$$
\kappa_{0}(s)=\sum_{i=1}^{m} w_{i} \Phi_{i}(s),
$$

where the $\Phi_{i}$ 's are known continuous basis functions, the unknown weights $w_{i}$ are independent of $s$ and lie in known intervals of the form $\left(\underline{w}_{i}, \bar{w}_{i}\right)$ for $i=1,2, \ldots, m$ with con- 
stant endpoints, and $m \geq 1$; see Zhang et al. (1998) for background on artificial neural networks. We again assume that we know constant upper and lower bounds for the curvature, i.e., constants $\bar{c}$ and $\underline{c}<\bar{c}$ such that $\kappa_{0}(s) \in(\underline{c}, \bar{c})$ for all $s \geq 0$. The result of this section ensures the convergence condition $\lim _{t \rightarrow \infty}(\rho(t), \phi(t))=\left(\rho_{0}, 0\right)$, even if the curvature is nonconstant, which is a stronger conclusion about $(\rho, \phi)$ than the ISS condition from Section 5 .

To prove the result, we define $U, \mathcal{S}$, and $\bar{\Delta}$ as in Theorem 1 , we use the $m$ dynamic extensions

$$
\dot{\hat{w}}_{i}=\left(\hat{w}_{i}-\underline{w}_{i}\right)\left(\bar{w}_{i}-\hat{w}_{i}\right) \frac{\cos (\phi) \Phi_{i}(s)}{\left(1+\left(\rho-\rho_{0}\right) \hat{\kappa}(s, t)\right)^{2}} \frac{\partial U}{\partial \phi}(\rho, \phi)
$$

having the state space $\left(\underline{w}_{i}, \bar{w}_{i}\right)$ for $i=1, \ldots, m$, where

$$
\hat{\kappa}_{0}(s, t)=\sum_{i=1}^{m} \hat{w}_{i}(t) \Phi_{i}(s),
$$

and we define the adaptive controller $u_{2}$ from (15) except with $\hat{\kappa}_{0}$ now depending on time $t$ and the arc length $s$. However, we continue our convention of omitting arguments of functions, when this would not lead to confusion.

Finally, we assume that the known upper and lower bounds $\bar{w}_{i}$ and $w_{i}$ for the weights $w_{i}$ in the curvature are such that (60) stays in the interval $(\underline{c}, \bar{c})$. Since the unknown parameters are the weights $w_{i}$ instead of the curvatures $\kappa(s)$ themselves, we use the new augmented errors $(\tilde{q}, \tilde{w})=$ $\left(\tilde{q}_{1}, \tilde{q}_{2}, \tilde{w}\right)=\left(\rho-\rho_{0}, \phi, \hat{w}_{1}-w_{1}, \ldots, \hat{w}_{m}-w_{m}\right)$, which has the same augmented error dynamics we gave in (16), except we must now replace the $\tilde{\kappa}_{0}$ error dynamics by

$$
\dot{\tilde{w}}_{i}=\frac{\left(w_{i}+\tilde{w}_{i}-\underline{w}_{i}\right)\left(\bar{w}_{i}-w_{i}-\tilde{w}_{i}\right) \Phi_{i}(s) \cos \left(\tilde{q}_{2}\right)}{\left(1+\left(\rho-\rho_{0}\right) \hat{\kappa}_{0}\right)^{2}} \frac{\partial U}{\partial \phi}(\rho, \phi)
$$

for $i=1, \ldots, m$, and $\tilde{\kappa}_{0}=\hat{\kappa}_{0}-\kappa_{0}$ now takes the form

$$
\tilde{\kappa}_{0}=\sum_{i=1}^{m} \tilde{w}_{i} \Phi_{i} .
$$

With these changes and $\mathcal{S}^{\sharp}$ from Theorem 1 as before, we can then prove the following (but see Remark 2 for cases where we can also identify the curvatures and prove ISS):

Theorem 3 Let the assumptions of Theorem 1 hold with $\bar{\delta}=0$. Then for all solutions $(\tilde{q}(t), \tilde{w}(t))=(\rho(t)-$ $\left.\rho_{0}, \phi(t), \hat{w}_{1}(t)-w_{1}, \ldots, \hat{w}_{m}(t)-w_{m}\right)$ of the augmented error dynamics that start in $\mathcal{S}_{w}^{\sharp}=\left\{(\tilde{q}, \tilde{w}): \tilde{q}+\left(\rho_{0}, 0\right) \in\right.$ $\mathcal{S}, \tilde{w}_{i} \in\left(\underline{w}_{i}-w_{i}, \bar{w}_{i}-w_{i}\right)$ for all $\left.i\right\}$, the corresponding solutions $\left(\tilde{\tilde{q}}(t), \tilde{\kappa}_{0}(t)\right)=\left(\rho(t)-\rho_{0}, \phi(t), \tilde{\kappa}_{0}(t)\right)$ remain in $\mathcal{S}^{\sharp}$ for all $t \geq 0$ and are such that $\lim _{t \rightarrow \infty}(\rho(t), \phi(t))=\left(\rho_{0}, 0\right)$.

Proof: We indicate the changes needed in the proof of Theorem 1. Lemma 3 and its proof remain the same, except with $\tilde{\kappa}$ and $\hat{\kappa}$ also depending on $s$. Then we change the augmented Lyapunov function to the function $U^{\sharp}$ : $\mathcal{S} \times \prod_{i=1}^{m}\left(\underline{w}_{i}-w_{i}, \bar{w}_{i}-w_{i}\right) \rightarrow[0, \infty)$ that is defined by

$$
\begin{aligned}
& U^{\sharp}(\rho, \phi, \tilde{w})= \\
& U(\rho, \phi)+\sum_{i=1}^{m} \int_{0}^{\tilde{w}_{i}} \frac{\ell}{\left(w_{i}+\ell-\underline{w}_{i}\right)\left(\bar{w}_{i}-w_{i}-\ell\right)} \mathrm{d} \ell
\end{aligned}
$$

where $U$ is from (6). The proof is then the same as the proof of Theorem 1 (with $\delta=0$ ) up through and including (29), with the same constant $\beta_{0}>0$. This gives

$$
\begin{aligned}
& \int_{0}^{t}\left[\left(h^{\prime}(\rho(\ell)) \cos (\phi(\ell))\right)^{2}+\sin ^{2}(\phi(\ell))\right] \mathrm{d} \ell \\
& \leq \frac{1}{\beta_{0}} U^{\sharp}(\rho(0), \phi(0), \tilde{w}(0))
\end{aligned}
$$

along all trajectories of the closed loop augmented error dynamics contained in $\mathcal{S}^{\sharp}$. By the forward invariance of $\mathcal{S}^{\sharp}$ from Lemma 3 , it follows that the function $\mathcal{L}(\ell)=$ $\left(h^{\prime}(\rho(\ell)) \cos (\phi(\ell))\right)^{2}+\sin ^{2}(\phi(\ell))$ is uniformly continuous on $\mathcal{S}$. Hence, the result follows from Barbalat's Lemma. $\square$

Remark 2 We can use the scaling method from Section 6 to enlarge the bounds on the curvatures $\kappa_{0}(s)$. Also, when the assumptions of Theorem 3 hold with $m=1$, we can use a variant of the last part of the proof of Theorem 1 to prove that $\lim _{t \rightarrow \infty} \hat{\kappa}_{0}(s, t)=\kappa_{0}(s)$ for all $s$, i.e., we get identification of the nonconstant curvatures. This is done by replacing $V^{\sharp}$ from (30) by

$$
V^{\sharp}\left(\tilde{q}, \tilde{w}_{1}\right)=\overline{\mathcal{M}}_{4} U^{\sharp}\left(\rho, \phi, \tilde{w}_{1}\right)+\tilde{q}_{2} \tilde{w}_{1}
$$

for a suitable constant $\overline{\mathcal{M}}_{4}>0$ and assuming that $\Phi_{1}(s)$ is bounded and admits a uniform positive lower bound $\underline{\Phi}>0$, i.e., $\Phi_{1}(s) \geq \underline{\Phi}$ for all $s \geq 0$. Here $U^{\sharp}$ is as defined in (63). To see how this can be done when $\delta=0$, first notice that Lemma 3 ensures that we have a constant $\overline{\mathcal{G}}_{1}^{*}>0$ such that

$$
\left|\dot{\tilde{w}}_{1}\right| \leq \overline{\mathcal{G}}_{1}^{*}|\tilde{q}| \text { and } U^{\sharp}\left(\rho, \phi, \tilde{w}_{1}\right) \geq \frac{\left|\left(\tilde{q}, \tilde{w}_{1}\right)\right|^{2}}{\mathcal{G}_{1}^{*}}
$$

on $\mathcal{S}^{\sharp}$. This gives positive constants $\overline{\mathcal{G}}_{2}^{*}$ and $\overline{\mathcal{G}}_{3}^{*}$ such that

$$
\begin{aligned}
\frac{d}{d t}\left(\tilde{q}_{2} \tilde{w}_{1}\right) \leq & \left(h^{\prime}\left(\tilde{q}_{1}+\rho_{0}\right) \cos \left(\tilde{q}_{2}\right)-\mu \sin \left(\tilde{q}_{2}\right)\right. \\
& \left.-\frac{\tilde{w}_{1} \cos (\phi) \Phi_{1}(s)}{\left(1+\kappa_{0}\left(\rho-\rho_{0}\right)\right)\left(1+\hat{\kappa}_{0}\left(\rho-\rho_{0}\right)\right)}\right) \tilde{w}_{1}+\overline{\mathcal{G}}_{1}^{*}|\tilde{q}|^{2} \\
\leq & \overline{\mathcal{G}}_{2}^{*}\left(\left|\tilde{w}_{1}\right||\tilde{q}|+|\tilde{q}|^{2}\right)-\overline{\mathcal{G}}_{3}^{*} \tilde{w}_{1}^{2}
\end{aligned}
$$

holds on $\mathcal{S}^{\sharp}$. Then we can argue as in the last part of the proof of Theorem 1 (with the $\overline{\mathcal{G}}_{i}$ 's replaced by the corresponding $\overline{\mathcal{G}}_{i}^{*}$ 's, and $\tilde{\kappa}_{0}$ replaced by $\left.\tilde{w}_{1}\right)$ to prove that the dynamics for $\left(\tilde{q}, \tilde{w}_{1}\right)=\left(\rho-\rho_{0}, \phi, \hat{w}_{1}-w_{1}\right)$ are $G A S$ with respect to $\left(0, \mathcal{S}^{\sharp}\right)$. Moreover, since the preceding analysis is based on a Lyapunov functional design (instead of the Barbalat's lemma approach from the proof of Theorem 3), we can extend it to cases with additive uncertainties $\delta$.

\section{Examples and Simulations}

To illustrate the value of our tracking, parameter identification, and robust forward invariance approach, first consider the special case of Theorem 2 where $h(\rho)=\left(\rho-\rho_{0}\right)^{2}$, $\rho_{0}=1, \rho_{*}=0.25, K=5 / 4, L=0.4$, and $\mu \in(0, \pi)$ is close enough to $\pi$. Here we use the fact that the right limit requirement $\lim _{\rho \rightarrow 0^{+}} h(\rho)=\infty$ from Assumption 1 can be omitted; see Section 5. Then our condition (10) holds, because we have

$$
\begin{aligned}
& \mu \tan \left(\mu \rho_{*}\right) \geq(0.9 \pi)(0.9)>2.5 \\
& >\max \left\{\left|h^{\prime}(\rho)\right|: \rho_{*} \leq \rho \leq \rho_{*}+K \rho_{0}\right\} \\
& =2 \max \{|\rho-1|: 0.25 \leq \rho \leq 1.5\}=1.5
\end{aligned}
$$


Also, the $\rho$ values occurring for points in $\mathcal{S}$ are in $[0.25,1.5]$, the $\phi$ values occurring in $\mathcal{S}$ are in $[-\pi / 4, \pi / 4]$, and we can choose the constant function $\gamma(\ell)=7.9$, the function $\Gamma(\ell)=4 \ell$, and the constants $\overline{\mathcal{M}}_{1}=5.4$ and $\overline{\mathcal{M}}_{2}=0.5$.

To compute the allowable constants $\bar{\Delta}$ for our perturbation bounds, notice that in terms of our earlier notation, we have $\Delta_{*}=\min \left\{\left|h^{\prime}(\rho) \cos (\phi)\right|:(\rho, \phi)^{\top} \in \mathrm{AB} \cup \mathrm{ED}\right\} \geq$ $2 \min \{|\rho-1| / \sqrt{2}: \rho \in[0.25,0.5] \cup[1.25,1.5]\} \approx 0.4$ and $\Delta_{* *}=\min \left\{\left|h^{\prime}(\rho) \cos (\phi)-\mu \sin (\phi)\right|:(\rho, \phi)^{\top} \in \mathrm{BC} \cup\right.$ $\mathrm{EF}\} \approx(1 / \sqrt{2}) \min \{|2(\rho-1)-0.9 \pi|: \rho \in[0.25,1.5]\} \approx$ $\sqrt{2} \min \{|\rho-2.4|: \rho \in[0.25,1.5]\} \approx 1$.3. Hence, we choose $\bar{\Delta}=0.4$. We also choose $\lambda=1 / 2$ and the disturbance bound $\bar{\delta}=2.5$. Since $\underline{c}=0$, we can omit $1 /\left(\rho_{*}+(K-1) \rho_{0}\right)$ from (51)-(52); see Remark 1 from Section 5. Then our lower bound on $\mathcal{M}_{*}$ from (50) is approximately 13.3.

Hence, since the desired distance to the curve is $\rho_{0}=1$, the upper bound on $\kappa_{0}$ provided by Theorem 2 is

$$
\kappa_{0}=\frac{\kappa}{1+\kappa}<\bar{c}<\frac{2}{3},
$$

which corresponds to allowing all constant curvatures $\kappa \in$ $(0,2)$. If we had instead used Theorem 1, e.g., with $\lambda=7 / 8$, then (18)-(19) with $\underline{c}=0$ would have produced the curvature bound $\bar{c}=0.2$. Therefore, we tripled the bound while also allowing control uncertainties $\delta:[0, \infty) \rightarrow[-2.5,2.5]$. Also, if we allow nonconstant curvatures, such as curvatures of the form $\kappa^{\sharp}(s)=\kappa+\eta(s)$ from Section 5 , then Section 5 ensures ISS with respect to the perturbation $\delta(t)$ from $(39)$, provided $\kappa \in(0,2)$ and provided that $|\delta(t)|<$ $\mathcal{M}_{*} \bar{\Delta} \approx 5.32$, which holds if $\sup _{s}|\eta(s)|<5.32$; see (39).

We simulated (49), using Mathematica and the preceding choices of the parameters and functions, so $\bar{\delta}=2.5$, the scaling constant is $\mathcal{M}_{*}=13.3$, and the curvature bound for $\bar{c}$ is $2 / 3$. We provide simulations for the cases $\delta(t)=0$ and then $\delta(t)=2.5 \sin (t)$ (where $\delta$ can represent control uncertainty, or the effects (39) of the nonconstant part $\eta(t)$ of the curvature as in the previous paragraph). In our first two simulations, we took $\kappa_{0}=0.5$ (corresponding to the unscaled curvature $\kappa=\kappa_{0} /\left(1-\rho_{0} \kappa_{0}\right)=1$, using (13)), the scaling constant $\mathcal{M}_{*}=13.3$, and the initial value $\left(\tilde{q}(0), \tilde{\kappa}_{0}(0)\right)=(-0.7, \pi / 4,0.15)$ for the augmented error variable, which corresponds to taking the initial value $(\rho(0), \phi(0))$ for the state to be

$$
B=\left(\rho_{*}\left(1+\frac{1}{\mathcal{M}_{*}}\right), \mu \rho_{*}\right) \approx(0.3,0.8)
$$

of the corresponding robustly forwardly invariant hexagon and the initial value for the estimated scaled curvature to be $\hat{\kappa}_{0}(0)=\tilde{\kappa}_{0}(0)+\kappa_{0}=0.15+0.5=0.65$, i.e., $30 \%$ above the true scaled curvature value $\kappa_{0}=0.5$. Using the relationship (13) between $\kappa$ and $\kappa_{0}$, this corresponds to initializing the estimate $\hat{\kappa}$ of the unscaled curvature at

$$
\hat{\kappa}(0)=\frac{\hat{\kappa}_{0}(0)}{1-\rho_{0} \hat{\kappa}_{0}(0)} \approx 1.9,
$$

i.e., approximately twice the actual unscaled curvature value $\kappa=1$. Figures 2 and 3 show the corresponding plots for $\tilde{q}_{1}(t)=\rho(t)-\rho_{0}, \tilde{q}_{2}(t)=\phi(t)$, and the estimate $\hat{\kappa}(t)$.

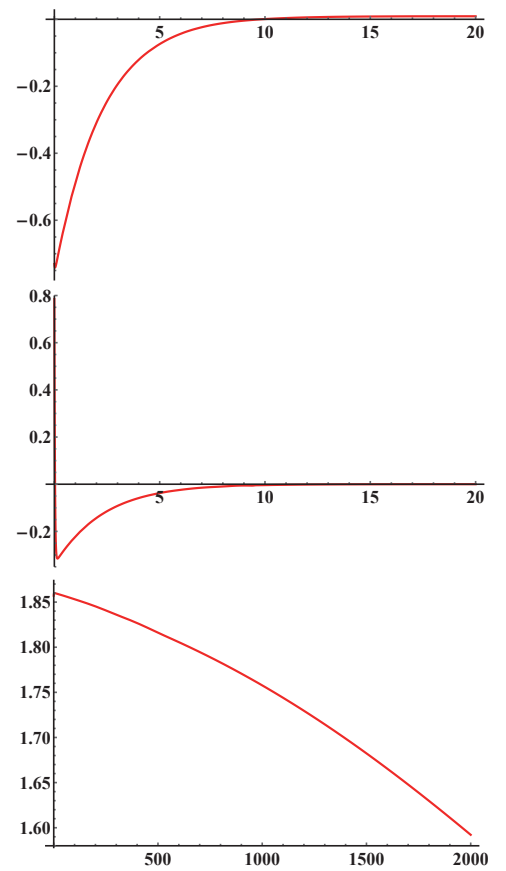

Fig. 2. Plots from Augmented Error Dynamics (49) with Initial Value $\left(\tilde{q}(0), \tilde{\kappa}_{0}(0)\right)=(-0.7, \pi / 4,0.15)$ and $\delta=0$. From Top to Bottom, $\tilde{q}_{1}(t)=\rho(t)-\rho_{0}, \tilde{q}_{2}(t)=\phi(t)$, and $\hat{\kappa}(t)$.

In our third simulation, we took the same parameters and disturbance function $\delta(t)=2.5 \sin (t)$ as in our second simulation, except we changed the initial value $(\rho(0), \phi(0))$ for the state to be the bottom right vertex

$$
E=\left(K \rho_{0}+\left(1-\frac{1}{\mathcal{M}_{*}}\right) \rho_{*},-\mu \rho_{*}\right) \approx(1.5,-0.8)
$$

of the corresponding robustly forwardly invariant hexagon. We report results for our third simulation in Fig. 4, which shows convergence of the state errors towards 0 , and the parameter identifiers converging to 1 , while staying in the robustly forwardly invariant set $\mathcal{S}^{\sharp}$, with overshoots from our perturbation in Figs. 3-4. Fig. 5 shows $(\rho(t), \phi(t))=$ $\left(\tilde{q}_{1}(t)+\rho_{0}, \tilde{q}_{2}(t)\right)$ staying in the corresponding set $\mathcal{S}$ for all three simulations. This agrees with our theory.

\section{Conclusions}

Adaptive planar curve tracking under unknown curvatures is important for the control of robots in uncertain environments. While our works Malisoff and Zhang (2013) and Malisoff and Zhang (2015) solve adaptive tracking and parameter identification problems under unknown control gains but known curvatures, here we solved a complementary problem, where the control gains are known but where our adaptive controller can cope with unknown curvatures. Our strict Lyapunov function approach allows us to prove input-to-state stability and to cover nonconstant curvatures, and our robust forward invariance approach makes it possible to satisfy a valuable class of polygonal state constraints. One can study even more general threedimensional cases with input delays, and identify uncertain control gains and uncertain curvatures, using estima- 


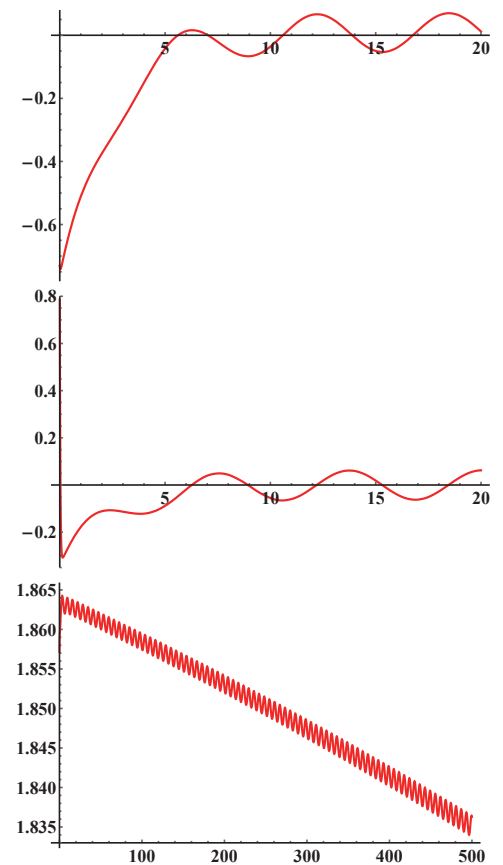

Fig. 3. Plots from Augmented Error Dynamics (49) with Initial Value $\left(\tilde{q}(0), \tilde{\kappa}_{0}(0)\right)=(-0.7, \pi / 4,0.15)$ and $\delta(t)=2.5 \sin (t)$. From Top to Bottom, $\tilde{q}_{1}(t)=\rho(t)-\rho_{0}, \tilde{q}_{2}(t)=\phi(t)$, and $\hat{\kappa}(t)$.

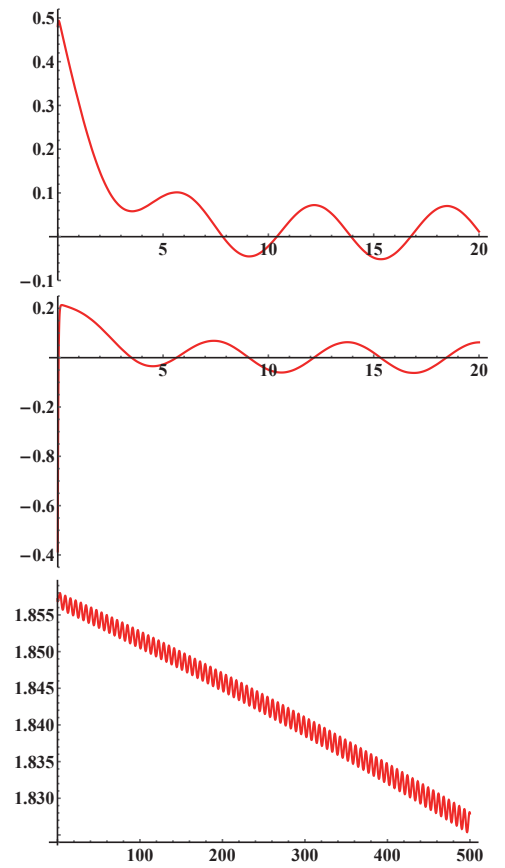

Fig. 4. Plots from Augmented Error Dynamics (49) with Initial Value $\left(\tilde{q}(0), \tilde{\kappa}_{0}(0)\right)=(0.5,-\pi / 4,0.15)$ and $\delta(t)=2.5 \sin (t)$. From Top to Bottom, $\tilde{q}_{1}(t)=\rho(t)-\rho_{0}, \tilde{q}_{2}(t)=\phi(t)$, and $\hat{\kappa}(t)$.

tors for the unknown curvatures and the unknown control gains. We leave our generalizations for a future work.

\section{References}

Aguiar, A. and J. Hespanha (2007). Trajectory-tracking and path-following of underactuated autonomous vehi-

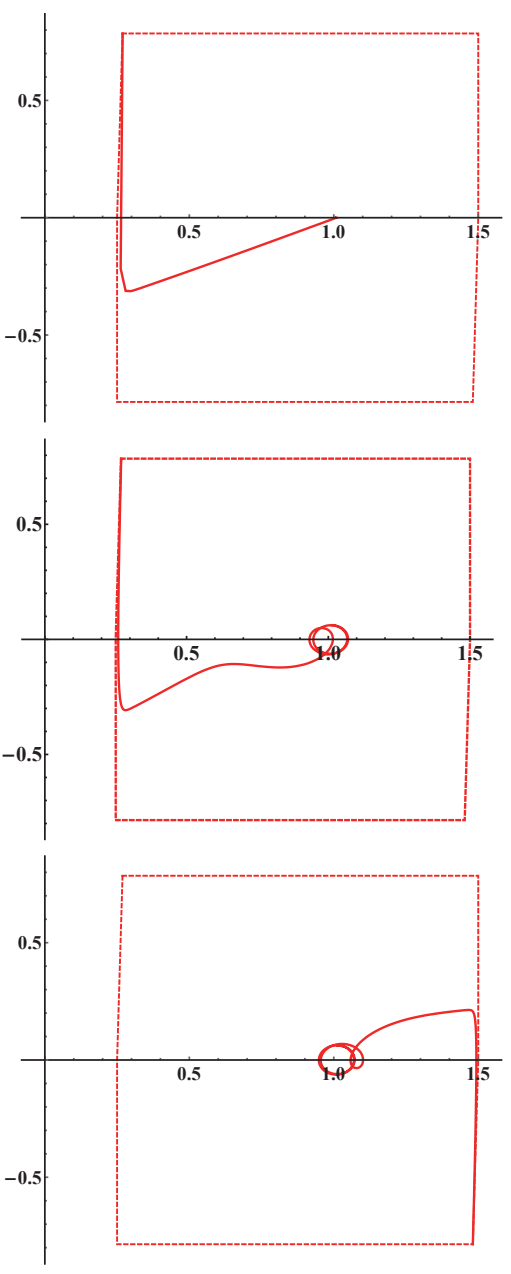

Fig. 5. States $(\rho(t), \phi(t))=\left(\tilde{q}_{1}(t)+\rho_{0}, \tilde{q}_{2}(t)\right)$ Staying in Robustly Forwardly Invariant Set on Time Interval $[0,20]$ Without Disturbances (Top) and $\delta(t)=2.5 \sin (t)$ (Middle and Bottom).

cles with parametric modeling uncertainty. IEEE Transactions on Automatic Control 52(8), 1362-1379.

Borhaug, E. and K. Pettersen (2005). Adaptive way-point tracking control for underactuated autonomous vehicles. In Proc. 44th IEEE Conf. on Decision and Control. Seville, Spain. pp. 4028-4034.

Bresch-Pietri, D. and M. Krstic (2010). Delay-adaptive predictor feedback for systems with unknown long actuator delay. IEEE Transactions on Automatic Control 55(9), 2106-2112.

Bresch-Pietri, D. and M. Krstic (2014). Delay-adaptive control for nonlinear systems. IEEE Transactions on Automatic Control 59(5), 1203-1218.

Do, K. and J. Pan (2006). Robust path-following of underactuated ships: Theory and experiments on a model ship. Ocean Engineering 33(10), 1354-1372.

Do, K., Z.P. Jiang and J. Pan (2004). Robust adaptive path following of underactuated ships. Automatica 40(6), 929-944.

Justh, E. and P. Krishnaprasad (2005). Natural frames and interacting particles in three dimensions. In Proc. 45th IEEE Conf. on Decision and Control and European 
Control Conf.. San Diego, CA. pp. 2841-2846.

Khalil, H. (2002). Nonlinear Systems, Third Edition. Prentice Hall. Upper Saddle River, NJ.

Krstic, M. (2009). Delay Compensation for Nonlinear, Adaptive, and PDE Systems. Birkhauser. Boston, MA.

Lenain, R., B. Thuilot, C. Cariou and P. Martinet (2006). High accuracy path tracking for vehicles in presence of sliding: Application to farm vehicle automatic guidance for agricultural tasks. Autonomous Robots 21(1), 79-97.

Lumelsky, V. and A. Stepanov (1987). Path planning strategies for a point mobile automaton moving amidst unknown obstacles of arbitrary shape. Algorithmica 2(2), 403-430.

Malisoff, M. and F. Zhang (2013). Adaptive control for planar curve tracking under controller uncertainty. Automatica 49(5), 1411-1418.

Malisoff, M. and F. Zhang (2015). Robustness of adaptive control under time delays for three-dimensional curve tracking. SIAM Journal on Control and Optimization 53(4), 2203-2236.

Malisoff, M. and F. Zhang (2016). Adaptive planar curve tracking control with unknown curvature. In Proc. American Control Conf.. Boston, MA. pp. 1608-1612.

Malisoff, M., F. Mazenc and F. Zhang (2012). Stability and robustness analysis for curve tracking control using input-to-state stability. IEEE Transactions on Automatic Control 57(5), 1320-1326.

Micaelli, A. and C. Samson (1993). Trajectory tracking for unicycle-type and two-steering-wheels mobile robots. INRIA Report $209 \%$.

Morin, P. and C. Samson (2008). Motion control of wheeled mobile robots. In Springer Handbook of Robotics. Springer. Berlin, Germany. pp. 799-826.

Mukhopadhyay, S., C. Wang, M. Patterson, M. Malisoff and F. Zhang (2014). Collaborative autonomous surveys in marine environments affected by oil spills. In Cooperative Robots and Sensor Networks. Springer. New York. pp. $87-113$.

Woolsey, C. and L. Techy (2009). Cross-track control of a slender, underactuated AUV using potential shaping. Ocean Engineering 36(1), 82-91.

Zhang, F., A. O'Connor, D. Luebke and P. Krishnaprasad (2004a). Experimental study of curvature-based control laws for obstacle avoidance. In Proc. IEEE International Conf. on Robotics and Automation. New Orleans, LA. pp. 3849-3854.

Zhang, F., D. Fratantoni, D. Paley, J. Lund and N. Leonard (2007). Control of coordinated patterns for ocean sampling. International Journal of Control 80(7), 11861199.

Zhang, F., E. Justh and P. Krishnaprasad (2004b). Boundary following using gyroscopic control. In Proc. IEEE Conf. on Decision and Control. Paradise Island, Bahamas. pp. 5204-5209.

Zhang, F., J. Chen, Z. Wang and Y. Han (1998). Fault recognition of a 20 mvar statcom main circuit using artificial neural network. In Proc. 33rd Intersociety Energy Conversion Engineering Conf.. Colorado Springs, CO. 